\title{
A CONVERGENT SCHEME FOR A NON-LOCAL COUPLED SYSTEM MODELLING DISLOCATIONS DENSITIES DYNAMICS
}

\author{
A. EL HAJJ AND N. FORCADEL
}

\begin{abstract}
In this paper, we study a non-local coupled system that arises in the theory of dislocations densities dynamics. Within the framework of viscosity solutions, we prove a long time existence and uniqueness result for the solution of this model. We also propose a convergent numerical scheme and we prove a Crandall-Lions type error estimate between the continuous solution and the numerical one. As far as we know, this is the first error estimate of Crandall-Lions type for Hamilton-Jacobi systems. We also provide some numerical simulations.
\end{abstract}

\section{INTRODUCTION}

1.1. Presentation and physical motivations. A dislocation is a crystal defect which corresponds to a discontinuity in the crystalline structure organisation. This concept was introduced by Polanyi, Taylor and Orowan in 1934 as the main explanation at the microscopic scale of plastic deformation. A dislocation creates around it a perturbation that can be seen as an elastic field. Under an exterior strain, a dislocation moves according to its Burgers vector which characterizes the intensity and the direction of the defect displacement (see Hirth and Lothe [17 for an introduction to dislocations).

Here, we are interested in dislocations densities dynamics. More precisely, we consider edge dislocations, i.e., the Burgers vectors and dislocations are in the same plane. These dislocations are moving with the Burgers vectors $\pm \vec{b}$ (see Figure 1). This model was introduced by Groma and Balogh as a coupled system, namely a transport problem where the velocity is given by the elasticity equations in the $2-\mathrm{D}$ case (see [16]).

If the 2-D domain is 1-periodic in $x_{1}$ and $x_{2}$, and if the dislocations densities depend only on the variable $x=x_{1}+x_{2}$ (where $\left(x_{1}, x_{2}\right)$ are the coordinates of a point in $\mathbb{R}^{2}$ ), when $\vec{b}=(1,0)$, the 2 -D model of [16] reduces to the system of

Received by the editor June 15, 2006 and, in revised form, January 26, 2007.

2000 Mathematics Subject Classification. Primary 35Q72, 49L25, 35F25, 35L40, 65M06, $65 \mathrm{M} 12,65 \mathrm{M} 15,74 \mathrm{H} 20,74 \mathrm{H} 25$.

Key words and phrases. Hamilton Jacobi equations, viscosity solutions, dislocations densities dynamics, numerical scheme, error estimate, system.

(C)2007 American Mathematical Society Reverts to public domain 28 years from publication 


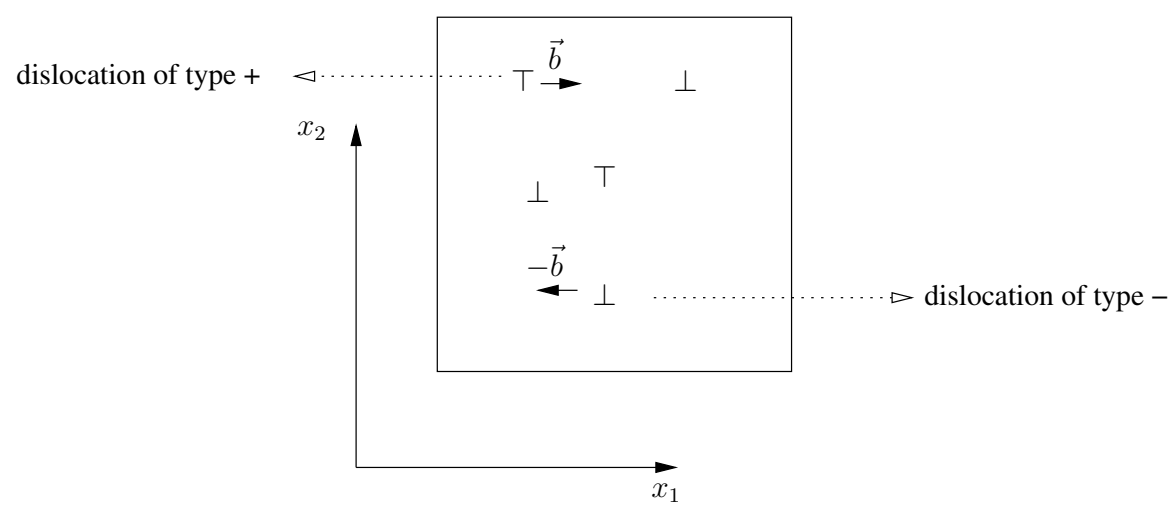

FiguRE 1. The cross-section of the dislocations lines.

coupled 1-D non-local Hamilton-Jacobi equations (see Section 2)

$$
\left\{\begin{array}{r}
\left(\rho_{+}\right)_{t}=-\left(\rho_{+}-\rho_{-}+\int_{0}^{1}\left(\rho_{+}(x, t)-\rho_{-}(x, t)\right) d x+L(t)\right)\left|D \rho_{+}\right| \\
\text {on } \mathbb{R} \times(0, T), \\
\left(\rho_{-}\right)_{t}=\left(\rho_{+}-\rho_{-}+\int_{0}^{1}\left(\rho_{+}(x, t)-\rho_{-}(x, t)\right) d x+L(t)\right)\left|D \rho_{-}\right| \\
\text {on } \mathbb{R} \times(0, T),
\end{array}\right.
$$

where $\rho_{+}, \rho_{-}$are the unknown scalars such that $\left(\rho_{+}-\rho_{-}\right)$represents the plastic deformation, their space derivatives $D \rho_{ \pm}:=\frac{\partial \rho_{ \pm}}{\partial x}$ are the dislocations densities and $L(t)$ represents the exterior shear stress field. From a physical viewpoint, $D \rho_{ \pm} \geq 0$, however, here we do not make this assumption to remain on a more general framework. The initial conditions for the system (1.1) are defined as

$$
\rho_{ \pm}(x, 0)=\rho_{ \pm}^{0}(x)=P_{ \pm}^{0}(x)+L_{0} x \text { on } \mathbb{R}
$$

where $P_{ \pm}^{0}$ are periodic of period 1 and Lipschitz continuous. In particular, $\rho_{+}^{0}-\rho_{-}^{0}$ is a 1-periodic function. $L_{0}$ is a given constant which is the total densities of type \pm , i.e., we suppose that initially, we have the same total density of type + and - .

1.2. Main results. The first goal of our paper is to prove the existence and uniqueness for the solution of the non-local system (1.1)-(1.2). A natural framework for our study is the viscosity solution theory. We refer to Barles [7, Bardi, Capuzzodolcetta [6] and Crandall, Ishii, Lions [11] for a good introduction to this theory in the scalar case. We also refer to Ishii, Koike [19] and Ishii [18 for the vectorial case and to Engler, Lenhart [12, Ishii, Koike [20], Lenhart [23], Lenhart, Belbas [24], Lenhart, Yamada 25] and Yamada 28] for some applications.

We have the following existence and uniqueness result for the non-local system:

Theorem 1.1 (Existence and uniqueness for the non-local problem). For all $T>0$, for all $L_{0} \in \mathbb{R}$, suppose that $\rho_{ \pm}^{0} \in \operatorname{Lip}(\mathbb{R})$ satisfy (1.2) and $L \in W^{1, \infty}\left(\mathbb{R}^{+}\right)$. Then, the system (1.1)-(1.2) admits a unique viscosity solution $\rho=\left(\rho_{+}, \rho_{-}\right)$. Moreover, this solution is uniformly Lipschitz continuous in space and time. 
Remark 1.2. If at initial time, we have $D \rho_{ \pm}^{0} \geq 0$, then this remains true for $t \geq 0$, i.e., $D \rho_{ \pm}(x, t) \geq 0$ for all $(x, t) \in \mathbb{R} \times[0, T]$. This allows us to treat the physical case where $D \rho_{ \pm} \geq 0$.

The main difficulty comes from the fact that the comparison principle does not hold because of the non-local term. In order to overcome this problem, we classically use a fixed point method by freezing the non-local term. In a first time, we give an existence and uniqueness result for the local problem (this is a simple adaptation of [19]). Then, we use Lipschitz estimates on the solution to prove the short time existence and uniqueness for the non-local system. In the third step, we obtained the result for all time by iterating the process.

Here, we are interested in the dislocations densities dynamics. Some others models have been proposed to describe the dynamics of dislocations lines. We recall some recent results. A non-local Hamilton-Jacobi equation was proposed by Alvarez, Hoch, Le Bouar and Monneau [5, 4] for modelling dislocation dynamics. They also proved a short time existence and uniqueness result for this model. We also refer to Alvarez, Cardaliaguet, Monneau [1] and Barles, Ley [8 for a long time result under certain monotony assumptions and to Forcadel 13 for a short time result for dislocations dynamics with a mean curvature term.

The second result is a numerical analysis of the non-local system (1.1). We propose a numerical scheme for our non-local system. Then, we give an error estimate between the continuous solution and the numerical one.

We want to approximate the solution of (1.1)-(1.2). Given a mesh size $\Delta x, \Delta t$, we define

$$
\Xi=\{i \Delta x, i \in \mathbb{Z}\} \quad \Xi_{T}=\Xi \times\left\{0, \ldots,(\Delta t) N_{T}\right\}
$$

where $N_{T}$ is the integer part of $T / \Delta t$. We refer generically to the lattice by $\Delta$ in the sequel. The discrete running point is $\left(x_{i}, t_{n}\right)$ with $x_{i}=i(\Delta x), t_{n}=n(\Delta t)$. We assume that $\Delta x+\Delta t \leq 1$. The approximation of the solution $\rho_{k}$ at the node $\left(x_{i}, t_{n}\right)$ is written indifferently as $v_{k}\left(x_{i}, t_{n}\right)$ or $v_{k, i}^{n}$ according to whether we view it as a function defined on the lattice or as a sequence.

Now, we will introduce the numerical scheme. The main difficulty is due to the non-local term, which requires the availability of the solution we intend to approximate. To solve this problem, we fix the solution $v_{i}^{n}=\left(v_{+, i}^{n}, v_{-, i}^{n}\right)$ at each time step on the interval $\left[t_{n}, t_{n+1}\right]$ and we apply the following monotone scheme,

$$
v_{i}^{0}=\left(v_{+, i}^{0}, v_{-, i}^{0}\right)=\tilde{\rho}^{0}\left(x_{i}\right)=\left(\tilde{\rho}_{+}^{0}, \tilde{\rho}_{-}^{0}\right),
$$

where $\tilde{\rho}_{ \pm}^{0}\left(x_{i}\right)$ is an approximation of $\rho_{ \pm}^{0}\left(x_{i}\right)$

$$
v_{k, i}^{n+1}=v_{k, i}^{n}+\Delta t C_{k}^{\Delta}[v]\left(x_{i}, t_{n}\right)\left\{\begin{array}{c}
E^{+}\left(D^{+} v_{k, i}^{n}, D^{-} v_{k, i}^{n}\right) \\
\text { if } C_{k}^{\Delta}[v]\left(x_{i}, t_{n}\right) \geq 0, \quad \forall k \in\{+,-\} \\
E^{-}\left(D^{+} v_{k, i}^{n}, D^{-} v_{k, i}^{n}\right) \\
\text { if not, }
\end{array}\right.
$$

where

$$
C_{k}^{\Delta}[v]\left(x_{i}, t_{n}\right)=-k\left(v_{+, i}^{n}-v_{-, i}^{n}+a^{\Delta}[v]\left(t_{n}\right)\right)
$$

and the non-local term $a^{\Delta}[v]\left(t_{n}\right)$ is given by

$$
a^{\Delta}[v]\left(t_{n}\right)=\sum_{i=0}^{N_{x}-1} \Delta x\left(v_{+}\left(x_{i}, t_{n}\right)-v_{-}\left(x_{i}, t_{n}\right)\right)+L\left(t_{n}\right)
$$


where $N_{x}$ is the integer part of $1 / \Delta x . E^{ \pm}$are the approximation of the Euclidean norm proposed by Osher and Sethian [26]:

$$
\begin{aligned}
& E^{+}(P, Q)=\left(\max (P, 0)^{2}+\min (Q, 0)^{2}\right)^{\frac{1}{2}}, \\
& E^{-}(P, Q)=\left(\min (P, 0)^{2}+\max (Q, 0)^{2}\right)^{\frac{1}{2}}
\end{aligned}
$$

and $D^{+} v_{k, i}^{n}, D^{-} v_{k, i}^{n}$ are the discrete gradients for all $n \in\left\{0, \ldots, N_{T}\right\}, i \in \mathbb{Z}$ and $k \in\{+,-\}$ :

$$
\begin{aligned}
& D^{+} v_{k, i}^{n}=\frac{v_{k, i+1}^{n}-v_{k, i}^{n}}{\Delta x}, \\
& D^{-} v_{k, i}^{n}=\frac{v_{k, i}^{n}-v_{k, i-1}^{n}}{\Delta x} .
\end{aligned}
$$

Finally, we assume the following uniform CFL condition (see the beginning of Section 5.2 for more details)

$$
\Delta t \leq \frac{1}{2 L_{2}} \Delta x
$$

where

$$
L_{2}=2 M+2
$$

with $M=\left\|P_{+}^{0}-P_{-}^{0}\right\|_{L^{\infty}(\mathbb{R})}$.

We then have the following error estimate:

Theorem 1.3 (Discrete-continuous error estimate). Let $T \geq 0$. Assume that $\Delta x+$ $\Delta t \leq 1, L \in W^{1, \infty}(\mathbb{R} \times[0, T))$ and that the $C F L$ condition (1.8) holds.

Then there exists a constant $K>0$ depending only on $\left\|P_{+}^{0}-P_{-}^{0}\right\|_{L^{\infty}(\mathbb{R})}$, $\max _{k \in\{+,-\}}\left\|D \rho_{k}^{0}\right\|_{L^{\infty}(\mathbb{R})}$ and $\|L\|_{W^{1, \infty}(0, T)}$ such that the error estimate between the continuous solution $\rho$ of the system (1.1)-(1.2) and the discrete solution $v$ of the finite difference scheme (1.3)-(1.4) is given by

$$
\max _{k \in\{+,-\}} \sup _{\Xi_{T}}\left|\rho_{k}-v_{k}\right| \leq K\left((T+\sqrt{T})(\Delta x+\Delta t)^{1 / 2}+\max _{k \in\{+,-\}} \sup _{\Xi}\left|\rho_{k}^{0}-v_{k}^{0}\right|\right),
$$

provided that

$$
K\left((T+\sqrt{T})(\Delta x+\Delta t)^{\frac{1}{2}}+\max _{k \in\{+,-\}} \sup _{\Xi}\left(\rho_{k}^{0}-v_{k}^{0}\right)\right) \leq 1 .
$$

Remark 1.4. In the condition

$$
K\left((T+\sqrt{T})(\Delta x+\Delta t)^{\frac{1}{2}}+\max _{k \in\{+,-\}} \sup _{\Xi}\left(\rho_{k}^{0}-v_{k}^{0}\right)\right) \leq 1,
$$

we can replace the right hand side by any positive constant.

In fact in the proof of this theorem, we mimic the continuous problem by considering the approximate solution of (1.1) as a fixed point of a local system. We are inspired by [3] to prove a Crandall-Lions rate of convergence [10, between the continuous solution of (1.1) and the numerical one. As far as we know, this is the first error estimate of Crandall-Lions type for Hamilton-Jacobi systems. We also refer to Jakobsen, Karlsen [21] and Jakobsen, Karlsen, Risebro [22] where they proved an error estimate for a weakly coupled system of the form

$$
\left(u_{i}\right)_{t}+H_{i}\left(t, x, u_{i}, D u_{i}\right)=G_{i}(t, x, u) \quad \text { in } \mathbb{R}^{N} \times(0, T)
$$


for $i=1, \ldots, M$. Their error estimate is in $O(\Delta t)$ for a semi-discrete splitting algorithm that they propose to approach the solution of (1.9). However, we obtain an error estimate in $O(\sqrt{\Delta t+\Delta x})$ because we also discretize in space.

In the dynamics of dislocations lines case, the model has also been numerically studied by Alvarez, Carlini, Monneau and Rouy [2, 3. In their paper, they proposed a numerical scheme for the non-local Hamilton-Jacobi equation and they proved a Crandall-Lions type rate of convergence.

Let us now explain how the paper is organized. We present the formal derivation of the model in Section 2. Then, in Section 3, we study the continuous problem. First in Subsection 3.1, we give an existence and uniqueness result for a local system. Then, in Subsection 3.2, we prove Theorem 1.1 by using a fixed point method. In Section 4, we prove a Crandall-Lions type error estimate for the local problem and then we prove Theorem 1.3 on the non-local problem. Some numerical examples are displayed in Section 5 where we show some tests illustrating our error estimate and then an evolution approximation of dislocation densities.

Notation. For simplicity of presentation, we fix some notations:

(1) Order relation: for $r=\left(r_{1}, r_{2}\right), s=\left(s_{1}, s_{2}\right) \in \mathbb{R}^{2}$, we say that $r \leq s$ if $r_{k} \leq s_{k}$ for $k \in\{1,2\}$.

(2) Addition vector-scalar: for $r=\left(r_{1}, r_{2}\right) \in \mathbb{R}^{2}, \lambda \in \mathbb{R}$, we denote by $r+\lambda$ the vector $\left(r_{1}+\lambda, r_{2}+\lambda\right)$.

(3) $P$-periodic plus $L_{0}$-linear function: we say that $\rho$ is $P$-periodic plus $L_{0^{-}}$ linear if there exists a vectorial periodic in space function $P^{\rho}=\left(P_{+}^{\rho}, P_{-}^{\rho}\right)$ of period $P$ and a constant $L_{0}$ such that

$$
\rho(x, t)=P^{\rho}(x, t)+L_{0} x=\left(P_{+}^{\rho}(x, t)+L_{0} x, P_{-}^{\rho}(x, t)+L_{0} x\right) .
$$

\section{Modelling}

We denote by $\mathbf{X}$ the vector $\mathbf{X}=\left(x_{1}, x_{2}\right)$. We consider a crystal with periodic deformation, namely the case where the total displacement of the crystal $U=$ $\left(U_{1}, U_{2}\right): \mathbb{R} \times \mathbb{R}^{+} \rightarrow \mathbb{R}^{2}$ can be decomposed in a 1-periodic displacement $u=\left(u_{1}, u_{2}\right)$ and a linear displacement $A(t)^{t} \mathbf{X}$ with $A(t)$ a given $2 \times 2$ matrix, which represents the shear stress

$$
A(t)=\left(\begin{array}{ll}
A_{11}(t) & A_{12}(t) \\
A_{21}(t) & A_{22}(t)
\end{array}\right) .
$$

The displacement $U$ is then given by

$$
U(\mathbf{X}, t)=u(\mathbf{X}, t)+A(t)^{t} \mathbf{X}
$$

and we define the total strain by

$$
\varepsilon(U)=\frac{1}{2}\left(\nabla U+{ }^{t} \nabla U\right)=\frac{1}{2}\left(\nabla u+{ }^{t} \nabla u+A(t)+{ }^{t} A(t)\right),
$$

where the coefficients of $\nabla u$ are $(\nabla u)_{i j}=\frac{\partial u_{i}}{\partial x_{j}}, i, j \in\{1,2\}$.

This total strain is decomposed in the form

$$
\varepsilon(U)=\varepsilon^{e}(U)+\varepsilon^{p},
$$

where $\varepsilon^{e}(U)$ is the elastic deformation and $\varepsilon^{p}$ is the plastic deformation, which is connected to the densities of dislocations by

$$
\varepsilon^{p}=\varepsilon^{0}\left(\rho_{+}-\rho_{-}\right),
$$


where $\rho_{ \pm}$represent the edge dislocation of type \pm , such that $\vec{b} . \nabla \rho_{ \pm} \geq 0$ is the density of dislocation of type,$\pm \vec{b}=\left(b_{1}, b_{2}\right)$ is the Burgers vector and

$$
\varepsilon^{0}=\frac{1}{2}\left(\vec{b} \otimes \vec{b}^{\perp}+\vec{b}^{\perp} \otimes \vec{b}\right)
$$

where $\vec{b}^{\perp}$ is a vector orthogonal to $\vec{b}$ and $\left(\vec{b} \otimes \vec{b}^{\perp}\right)_{i j}=b_{i} b_{j}^{\perp}$.

The stress is then given by

$$
\sigma=\Lambda: \varepsilon^{e}(U),
$$

i.e. the coefficients of the matrix $\sigma$ are

$$
\sigma_{i j}=\sum_{k, l \in\{1,2\}} \Lambda_{i j k l} \varepsilon_{k l}^{e}(U) \quad i, j \in\{1,2\}
$$

with $\Lambda=\left(\Lambda_{i j k l}\right)_{i j k l}, i, j, k, l=1,2, \Lambda_{i j k l}$ are the elastic constant coefficients of the material, satisfying for $m>0$,

$$
\sum_{i j k l=1,2} \Lambda_{i j k l} \varepsilon_{i j} \varepsilon_{k l} \geq m \sum_{i j=1,2} \varepsilon_{i j}^{2}
$$

for all symmetric matrix $\varepsilon=\left(\varepsilon_{i j}\right)_{i j}$ i.e. such that $\varepsilon_{i j}=\varepsilon_{j i}$.

The functions $\rho_{ \pm}$and $u$ are then solutions of the coupled system (see Groma, Balogh [16, [15] and Groma [14]):

$$
\left\{\begin{array}{llrl}
\operatorname{div} \sigma & =0 & & \text { in } \mathbb{R}^{2} \times(0, T), \\
\sigma & =\Lambda:\left(\varepsilon(U)-\varepsilon^{p}\right) & & \text { in } \mathbb{R}^{2} \times(0, T), \\
\varepsilon(U) & =\frac{1}{2}\left(\nabla u+{ }^{t} \nabla u+A(t)+{ }^{t} A(t)\right) & & \text { in } \mathbb{R}^{2} \times(0, T), \\
\varepsilon^{p} & =\varepsilon^{0}\left(\rho_{+}-\rho_{-}\right) & & \text {in } \mathbb{R}^{2} \times(0, T), \\
\left(\rho_{ \pm}\right)_{t} & = \pm\left(\sigma: \varepsilon^{0}\right) \vec{b} \cdot \nabla \rho_{ \pm} & & \text {in } \mathbb{R}^{2} \times(0, T),
\end{array}\right.
$$

i.e. in the coordinates

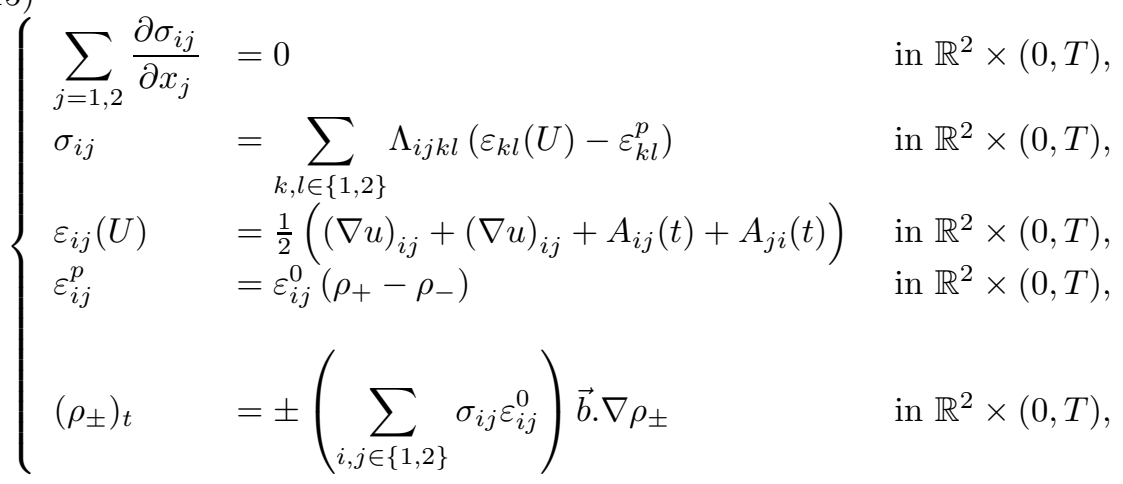

where the unknowns of the system are $\rho_{ \pm}$and the displacement $u=\left(u_{1}, u_{2}\right)$ and with $\varepsilon^{0}$ defined by (2.11). The sign \pm comes from \pm in $\pm \vec{b}$.

To simplify, we consider the homogeneous case. The coefficients $\Lambda_{i j k l}$ are such that

$$
\sigma=2 \mu \varepsilon^{e}(U)+\lambda \operatorname{tr}\left(\varepsilon^{e}(U)\right) I_{d}
$$


where $\mu>0$ and $\lambda+\mu>0$ (consequence of (2.13) ) are the Lamé coefficients and $I_{d}$ the identity matrix. Then, the following lemma holds:

Lemma 2.1 (Equivalence between 2-D and 1-D models). If we assume that the Burgers vector is $\vec{b}=(1,0)$, and that the densities of dislocations and $u$ only depend on one variable $x=x_{1}+x_{2}$ (as shown in Figure 2), the 2-D problem (2.14), with $\Lambda$ defined by (2.16) is equivalent to the 1-D problem

$$
\left\{\begin{array}{lll}
\left(\rho_{+}\right)_{t}=-C_{1}\left(\left(\rho_{+}-\rho_{-}\right)+C_{2} \int_{0}^{1}\left(\rho_{+}-\rho_{-}\right)+L(t)\right) D \rho_{+} & \text {in } \mathbb{R} \times(0, T), \\
\left(\rho_{-}\right)_{t}=C_{1}\left(\left(\rho_{+}-\rho_{-}\right)+C_{2} \int_{0}^{1}\left(\rho_{+}-\rho_{-}\right)+L(t)\right) D \rho_{-} & \text {in } \mathbb{R} \times(0, T),
\end{array}\right.
$$

where $L(t)=-\frac{(\lambda+2 \mu)}{(\lambda+\mu)}\left(A_{12}(t)+A_{21}(t)\right), C_{1}=\frac{(\lambda+\mu) \mu}{\lambda+2 \mu}$, and $C_{2}=\frac{\mu}{(\lambda+\mu)}$.

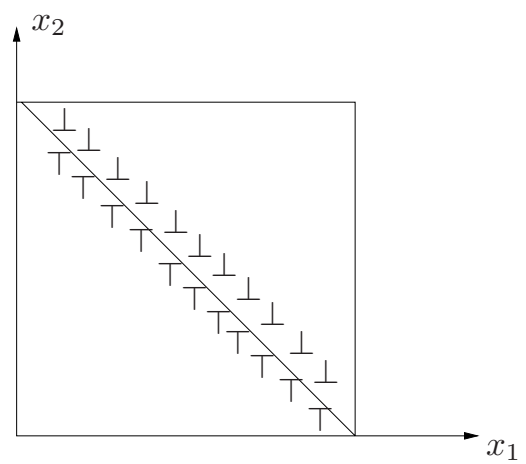

FiguRE 2. 1-D sub-model for invariance by translation in the direction $(-1,1)$

Proof of Lemma 2.1, We can rewrite the first equation of (2.14) and (2.16) as

$$
\operatorname{div}\left(2 \mu \varepsilon(U)+\lambda \operatorname{tr}(\varepsilon(U)) I_{d}\right)=\operatorname{div}\left(2 \mu \varepsilon^{p}+\lambda \operatorname{tr}\left(\varepsilon^{p}\right) I_{d}\right) .
$$

This implies by (2.10)

$$
\mu \Delta u+(\lambda+\mu) \nabla(\operatorname{div} u)=\mu\left(\begin{array}{c}
\frac{\partial\left(\rho_{+}-\rho_{-}\right)}{\partial x_{2}} \\
\frac{\partial\left(\rho_{+}-\rho_{-}\right)}{\partial x_{1}}
\end{array}\right) .
$$

Using the fact that $x=x_{1}+x_{2}$ yields

$$
2 \mu\left(\begin{array}{c}
\frac{\partial^{2} u_{1}}{\partial x^{2}} \\
\frac{\partial^{2} u_{2}}{\partial x^{2}}
\end{array}\right)+(\lambda+\mu)\left(\begin{array}{c}
\frac{\partial^{2}\left(u_{1}+u_{2}\right)}{\partial x^{2}} \\
\frac{\partial^{2}\left(u_{1}+u_{2}\right)}{\partial x^{2}}
\end{array}\right)=\mu\left(\begin{array}{c}
\frac{\partial\left(\rho_{+}-\rho_{-}\right)}{\partial x} \\
\frac{\partial\left(\rho_{+}-\rho_{-}\right)}{\partial x}
\end{array}\right) .
$$


Now, by adding the two equations above, we obtain

$$
\frac{\partial^{2}\left(u_{1}+u_{2}\right)}{\partial x^{2}}=\frac{\mu}{\lambda+2 \mu}\left(\frac{\partial\left(\rho_{+}-\rho_{-}\right)}{\partial x}\right) .
$$

Integrating the above equation yields, since $u$ is 1-periodic:

$$
\frac{\partial\left(u_{1}+u_{2}\right)}{\partial x}=\frac{\mu}{\lambda+2 \mu}\left(\left(\rho_{+}-\rho_{-}\right)-\int_{0}^{1}\left(\rho_{+}-\rho_{-}\right)\right) .
$$

Using the fact that

$$
\left(\sigma: \varepsilon^{0}\right)=\sigma_{12}=2 \mu\left(\varepsilon^{e}(U)\right)_{12}=\mu\left(\frac{\partial\left(u_{1}+u_{2}\right)}{\partial x}+A_{12}(t)+A_{21}(t)-\left(\rho_{+}-\rho_{-}\right)\right)
$$

and (2.18) yields

$$
\left(\sigma: \varepsilon^{0}\right)=-\frac{(\lambda+\mu) \mu}{\lambda+2 \mu}\left(\left(\rho_{+}-\rho_{-}\right)+\frac{\mu}{2(\lambda+\mu)} \int_{0}^{1}\left(\rho_{+}-\rho_{-}\right)+L(t)\right)
$$

where $L(t)=-\frac{(\lambda+2 \mu)}{(\lambda+\mu)}\left(A_{12}(t)+A_{21}(t)\right)$. We then deduce, if $\vec{b}=(1,0)$, that the system (2.14) can be rewritten as (2.17). As the constants $C_{1}, C_{2}$ are positive, to simplify the notations, we can put them into 1 in the following without loss of generality on the system (1.1).

\section{The COntinuous PROBlem}

To prove the existence and uniqueness result for the non-local problem, we use a fixed point method. In order to do that we freeze the non-local term and we study the following local problem:

$$
\left\{\begin{array}{lll}
\left(\rho_{+}\right)_{t}=-\left(\rho_{+}-\rho_{-}+a(t)\right)\left|D \rho_{+}\right| & \text {on } & \mathbb{R} \times(0, T), \\
\left(\rho_{-}\right)_{t}=\left(\rho_{+}-\rho_{-}+a(t)\right)\left|D \rho_{-}\right| & \text {on } & \mathbb{R} \times(0, T), \\
\rho_{+}(\cdot, 0)=\rho_{+}^{0} & \text { on } & \mathbb{R}, \\
\rho_{-}(\cdot, 0)=\rho_{-}^{0} & \text { on } & \mathbb{R} .
\end{array}\right.
$$

The assumptions are the following:

(H1) $a \in W^{1, \infty}\left(\mathbb{R}^{+}\right)$,

(H2) $\rho^{0}=\left(\rho_{+}^{0}, \rho_{-}^{0}\right)$ is 1-periodic plus $L_{0}$-linear, i.e., $\rho_{ \pm}^{0}(x)=P_{ \pm}^{0}(x)+L_{0} x$ where $P_{ \pm}^{0}$ are periodic of period 1 and $L_{0}$ is a constant.

(H3) $P_{ \pm}^{0} \in \operatorname{Lip}(\mathbb{R})$.

3.1. The local problem. Let $T>0$, we denote by $U S C(\mathbb{R} \times(0, T)$ ) (resp. $L S C(\mathbb{R} \times(0, T)))$ the set of locally bounded upper (resp. lower) semi-continuous functions. The key point is that our system is quasi-monotone in the sense of Ishii, Koike [19, (A.1)], (see Lemma 3.1 below) and so we can extend their results to our system in unbounded domain and with unbounded initial condition using the well-known arguments of the scalar case.

Lemma 3.1 (Quasi-monotony of the Hamiltonian). The Hamiltonian $H_{k}(t, \rho, p)=$ $k\left(\rho_{+}-\rho_{-}+a(t)\right)|p|$ is quasi-monotone, i.e., for all vectors $r$ and $s$ such that

$$
r_{j}-s_{j}=\max _{k \in\{+,-\}}\left(r_{k}-s_{k}\right) \geq 0
$$

then

$$
H_{j}(t, r, p)-H_{j}(t, s, p) \geq 0 .
$$


We then have the following theorem:

Theorem 3.2 (The local problem). Let $T \geq 0$. Assume (H1), (H2), (H3). We set $M=\left\|P_{+}^{0}-P_{-}^{0}\right\|_{L^{\infty}(\mathbb{R})}$ and $B_{0}=\max _{k \in\{+,-\}}\left\|D \rho_{k}^{0}\right\|_{L^{\infty}(\mathbb{R})}$. Then, the following holds:

(i) Comparison principle. Let $\rho \in U S C(\mathbb{R} \times(0, T))$ and $v \in L S C(\mathbb{R} \times$ $(0, T))$ be respectively sub- and super-solutions of (1.1)-1.2). We assume that there exists a constant $C>0$ such that

$$
\rho^{0}(x)-C t \leq \rho, v \leq \rho^{0}(x)+C t .
$$

If $\rho(\cdot, 0) \leq v(\cdot, 0)$ in $\mathbb{R}$, then $\rho \leq v$ in $\mathbb{R} \times[0, T]$.

(ii) Existence. There exists a unique viscosity solution $\rho$ of problem (3.19) satisfying

$$
\rho^{0}(x)-\left(M+\|a\|_{L^{\infty}(0, T)}\right) B_{0} t \leq \rho(x, t) \leq \rho^{0}(x)+\left(M+\|a\|_{L^{\infty}(0, T)}\right) B_{0} t .
$$

Moreover, the solution is 1-periodic plus $L_{0}$-linear.

(iii) Regularity. The solution $\rho$ of (3.19) is Lipschitz continuous in space and time and satisfies

$$
\begin{gathered}
\max _{k \in\{+,-\}}\left\|D \rho_{k}\right\|_{L^{\infty}(\mathbb{R} \times(0, T))} \leq B_{0}, \\
\max _{k \in\{+,-\}}\left\|\left(\rho_{k}\right)_{t}\right\|_{L^{\infty}(\mathbb{R} \times(0, T))} \leq B_{0}\left(M+\|a\|_{L^{\infty}(0, T)}\right) .
\end{gathered}
$$

(iv) Estimate on the solution. The solution $\rho$ satisfies

$$
\left\|\rho_{+}-\rho_{-}\right\|_{L^{\infty}(\mathbb{R} \times(0, T))} \leq\left\|\rho_{+}^{0}-\rho_{-}^{0}\right\|_{L^{\infty}(\mathbb{R})} .
$$

Proof of Theorem 3.2. The comparison principle is just an extension of the one of Ishii, Koike [19, Th. 4.7] for quasi-monotone Hamiltonians. For the existence, it suffices to use Perron's method by remarking that $\rho \pm\left(M+\|a\|_{L^{\infty}(0, T)}\right) B_{0} t$ are respectively super- and sub-solutions of (3.19). The fact that $\rho$ is 1-periodic plus $L_{0}$-linear comes from the fact that $\rho(x+1, t)+L_{0}$ is also solution of (3.19).

The Lipschitz estimate in space comes from the fact that problem (3.19) is invariant by space translation. To obtain the Lipschitz estimate in time, it is sufficient to bound the velocity using (3.22).

We now prove (iv). We set

$$
m_{+}(t)=\sup _{x \in(0,1)} \rho_{+}(x, t) \text { and } m_{-}(t)=\inf _{x \in(0,1)} \rho_{-}(x, t) .
$$

It is easy to check that $m_{+}$(resp. $m_{-}$) is a sub-solution (resp. super-solution) of $u_{t}=0$ which implies the upper bound of (iv). The lower bound is proved similarly. This ends the proof of the theorem.

3.2. The non-local problem. Before proving Theorem 1.1 we need the following lemma:

Lemma 3.3 (Stability of the solution with respect to the velocity). Let $T \geq 0$. We consider for $i=1,2$ two different equations,

$$
\begin{cases}\left(\rho_{k}^{i}\right)_{t}=-k\left(\rho_{+}^{i}-\rho_{-}^{i}+a_{i}(t)\right)\left|D \rho_{k}^{i}\right| & \text { for } \quad k \in\{+,-\}, \\ \rho_{k}^{i}(\cdot, 0)=\rho_{k}^{0} & \text { for } k \in\{+,-\},\end{cases}
$$


where the coefficients $a_{i}$ satisfy (H1) and the initial conditions $\rho^{0}=\left(\rho_{+}^{0}, \rho_{-}^{0}\right)$ satisfy (H2)-(H3). Then, we have

$$
\max _{k \in\{+,-\}}\left\|\rho_{k}^{2}-\rho_{k}^{1}\right\|_{L^{\infty}(\mathbb{R} \times(0, T))} \leq B_{0} T\left\|a_{2}-a_{1}\right\|_{L^{\infty}(0, T)},
$$

where $\rho^{i}$ for $i=1,2$ are the solutions of (3.23) given by Theorem 3.2 ,

Proof of Lemma 3.3. We set $K=\left\|a_{2}-a_{1}\right\|_{L^{\infty}(0, T)}$. We remark that $\rho^{2}$ is a subsolution of

$$
\left(\rho_{k}\right)_{t}+k\left(\rho_{+}-\rho_{-}+a_{1}(t)\right)\left|D \rho_{k}\right|-K B_{0}=0 .
$$

Moreover, $\rho^{1}+K B_{0} t$ is a solution of the same problem. By comparison principle, we then deduce

$$
\max _{k \in\{+,-\}}\left\|\rho_{k}^{2}-\rho_{k}^{1}\right\|_{L^{\infty}(\mathbb{R} \times(0, T))} \leq K B_{0} T .
$$

This is the estimate we want.

We have the following lemma whose proof is trivial:

Lemma 3.4 (Stability of the velocity a). Let $\rho^{1}, \rho^{2}$ be 1-periodic plus $L_{0}$-linear. We set $a\left[\rho^{i}\right](t)=\int_{0}^{1} \rho_{+}^{i}(x, t)-\rho_{-}^{i}(x, t) d x+L(t)$. Then the following holds:

$$
\left\|a\left[\rho^{2}\right]-a\left[\rho^{1}\right]\right\|_{L^{\infty}(0, T)} \leq 2 \max _{k \in\{+,-\}}\left\|\rho_{k}^{2}-\rho_{k}^{1}\right\|_{L^{\infty}(\mathbb{R} \times(0, T))} .
$$

We now prove Theorem 1.1.

Proof of Theorem 1.1. We define the set

$U_{T}=\left\{\begin{array}{l|l}\rho=\left(\begin{array}{c}\rho_{+} \\ \rho_{-}\end{array}\right) \in\left(L_{\text {Loc }}^{\infty}\right)^{2} \text {, s.t. } & \begin{array}{l}\left\|\rho_{+}-\rho_{-}\right\|_{L^{\infty}} \leq M \\ \rho \text { is 1-periodic plus } L_{0} \text {-linear } \\ \max _{k \in\{+,-\}}\left\|D \rho_{k}\right\|_{L^{\infty} \leq B_{0}} \\ \max _{k \in\{+,-\}}\left\|\left(\rho_{k}\right)_{t}\right\|_{L^{\infty}} \leq B_{0}\left(2 M+\|L\|_{L^{\infty}(0, T)}\right)\end{array}\end{array}\right\}$,

where $L_{0}$ is defined in $(H 2), B_{0}=\max _{k \in\{+,-\}}\left\|D \rho_{k}^{0}\right\|_{L^{\infty}(\mathbb{R})}$ and $M=\left\|P_{+}^{0}-P_{-}^{0}\right\|_{L^{\infty}(\mathbb{R})}$. For $\rho \in U_{T}$, we set

$$
a[\rho](t)=\int_{0}^{1} \rho_{+}(x, t)-\rho_{-}(x, t) d x+L(t) .
$$

We see that for any $\rho \in U_{T}, a[\rho]$ satisfies (H1) with $\|a[\rho]\|_{L^{\infty}(0, T)} \leq M+\|L\|_{L^{\infty}(0, T)}$.

For $\rho \in U_{T}$, we then define $v=G(\rho)=\left(G_{+}(\rho), G_{-}(\rho)\right)$ as the unique viscosity solution for $k=1,2$ (see Theorem 3.2) of

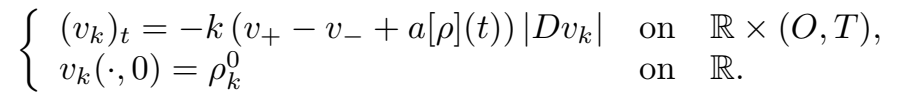

We will show that $G: U_{T} \rightarrow U_{T}$ is a strict contraction for $T$ small enough. First, we will prove that $G$ is well defined. By Theorem 3.2, we know that $v$ is 1-periodic plus $L_{0}$-linear. Moreover, we have

$$
\begin{gathered}
\max _{k \in\{+,-\}}\left\|D v_{k}\right\|_{L^{\infty}(\mathbb{R} \times(0, T))} \leq B_{0}, \\
\max _{k \in\{+,-\}}\left\|\left(v_{ \pm}\right)_{t}\right\|_{L^{\infty}(\mathbb{R} \times(0, T))} \leq B_{0}\left(M+\|a\|_{L^{\infty}(0, T)}\right) \leq B_{0}\left(2 M+\|L\|_{L^{\infty}(0, T)}\right)
\end{gathered}
$$


and

$$
\left\|v_{+}-v_{-}\right\|_{L^{\infty}(\mathbb{R} \times(0, T))} \leq M
$$

and so $v \in U_{T}$.

It thus remains to show that $G$ is a contraction. For $v^{i}=G\left(\rho^{i}\right)$, according to Lemma 3.3 and Lemma 3.4, we have

$$
\begin{aligned}
\left\|v^{2}-v^{1}\right\|_{L^{\infty}(\mathbb{R} \times(0, T))} & \sup _{\{k \in\{+,-\}\}}\left\|v_{k}^{2}-v_{k}^{1}\right\|_{L^{\infty}} \leq B_{0} T\left\|a\left[\rho^{2}\right]-a\left[\rho^{1}\right]\right\|_{L^{\infty}(0, T)} \\
& \leq 2 B_{0} T\left\|\rho^{1}-\rho^{2}\right\|_{L^{\infty}(\mathbb{R} \times(0, T))} \leq \frac{1}{2}\left\|\rho^{1}-\rho^{2}\right\|_{L^{\infty}(\mathbb{R} \times(0, T))}
\end{aligned}
$$

for $T \leq T^{*}=\frac{1}{4 B_{0}}$. And so $G$ is a contraction on $U_{T}$ which is a closed set. So, there exists a unique viscosity solution of (1.1)-(1.2) in $U_{T}$ on $\left(0, T^{*}\right)$ where $T^{*}=\frac{1}{4 B_{0}}$. By iterating this process, one can construct a solution for all $T>0$. Indeed, $T^{*}$ depends only on $B_{0}$ which does not change with time.

Proposition 3.5 (Estimate for the non-local solution). Let $T \geq 0$. The solution $\rho$ of (1.1)-(1.2) satisfies

$$
\left\|\rho_{+}-\rho_{-}\right\|_{L^{\infty}(\mathbb{R} \times(0, T))} \leq M
$$

where $M=\left\|P_{+}^{0}-P_{-}^{0}\right\|_{L^{\infty}(\mathbb{R})}$.

The proof is the same as the one for the local case; see Theorem 3.2 (iv).

\section{NumeriCAL SCHEME}

4.1. Approximation of the local system. In this subsection, we propose a finite difference scheme for the local system (3.19). Given a discrete velocity $a^{\Delta}$, we consider the discrete solution $v$ that approximates the solution of (3.19), given by the following explicit scheme

$$
v_{k, i}^{n+1}=v_{k, i}^{n}+\Delta t\left(C_{k}^{\Delta, \operatorname{Loc}}[v]\left(x_{i}, t_{n}\right)\right)\left\{\begin{array}{l}
E^{+}\left(D^{+} v_{k, i}^{n}, D^{-} v_{k, i}^{n}\right) \\
\quad \text { if } C_{k}^{\Delta, \operatorname{Loc}}[v]\left(x_{i}, t_{n}\right) \geq 0, \quad \forall k \in\{+,-\} \\
E^{-\left(D^{+} v_{k, i}^{n}, D^{-} v_{k, i}^{n}\right)} \\
\text { if not, }
\end{array}\right.
$$

where $\tilde{\rho}^{0}\left(x_{i}\right)$ are defined in (1.3),$E^{ \pm}$are the approximation of the Euclidean norm proposed by Osher and Sethian [26] defined in (1.6) (we also can use the one proposed by Rouy, Tourin [27]), $D^{+} v_{k}^{n}, D^{-} v_{k}^{n}$ are the discrete gradients defined in (1.7) and

$$
C_{k}^{\Delta, \operatorname{Loc}}[w]\left(x_{i}, t_{n}\right)=-k\left(w_{+}\left(x_{i}, t_{n}\right)-w_{-}\left(x_{i}, t_{n}\right)+a^{\Delta}\left(t_{n}\right)\right)
$$

where $a^{\Delta}$ is an approximation of $a$ satisfying

$$
a^{\Delta}\left(t_{n}\right)=a\left(t_{n}\right)
$$


In particular, the functions $E^{ \pm}$are Lipschitz continuous with respect to the discrete gradients, i.e.

$$
\left|E^{ \pm}(P, Q)-E^{ \pm}\left(P^{\prime}, Q^{\prime}\right)\right| \leq\left(\left|P-P^{\prime}\right|+\left|Q-Q^{\prime}\right|\right) .
$$

They are consistent with the Euclidean norm

$$
E^{ \pm}(P, P)=|P|
$$

and enjoy suitable monotonicity with respect to each variable

$$
\frac{\partial E^{+}}{\partial P^{+}} \geq 0, \quad \frac{\partial E^{+}}{\partial P^{-}} \leq 0, \quad \frac{\partial E^{-}}{\partial P^{+}} \geq 0, \quad \frac{\partial E^{-}}{\partial P^{-}} \leq 0 .
$$

Denoting by $S^{k}$ the operator on the right-hand side of (4.27), we can rewrite the scheme more compactly as

$$
v_{k, i}^{0}=\tilde{\rho}_{k}^{0}\left(x_{i}\right), \quad v_{k}^{n+1}=S^{k} v^{n} .
$$

Finally, we also assume that the mesh satisfies the following CFL condition (cf. Remark 4.2

$$
\Delta t \leq \frac{1}{2 L_{1}} \Delta x
$$

where

$$
L_{1}=\|a\|_{L^{\infty}(0, T)}+M+2 .
$$

Theorem 4.1 (Crandall-Lions rate of convergence). Let $T \leq 1$. Assume that $\Delta x+\Delta t \leq 1$. Assume that $a \in W^{1, \infty}(\mathbb{R} \times[0, T))$ and that the CFL condition (4.33) holds.

Then there exists a constant $K>0$ depending only on $\left\|P_{+}^{0}-P_{-}^{0}\right\|_{L^{\infty}(\mathbb{R})}$, $\max _{k \in\{+,-\}}\left\|D \rho_{k}^{0}\right\|_{L^{\infty}(\mathbb{R})}$ and $\|a\|_{W^{1, \infty}(0, T)}$ such that the error estimate between the continuous solution $\rho$ of the system (3.19) and the discrete solution $v$ of the finite difference scheme (4.26)-(4.27) is given by

$$
\begin{aligned}
& \max _{k \in\{+,-\}} \sup _{\Xi_{T}}\left|\rho_{k}\left(x_{i}, t_{n}\right)-v_{k, i}^{n}\right| \leq K \sqrt{T}(\Delta x+\Delta t)^{1 / 2}+\max _{k \in\{+,-\}} \sup _{\Xi}\left|\rho_{k}^{0}\left(x_{i}\right)-v_{k, i}^{0}\right| \\
& \text { provided } K(\Delta x+\Delta t)^{\frac{1}{2}}+\max _{k \in\{+,-\}} \sup _{\Xi}\left(\rho_{k}^{0}\left(x_{i}\right)-v_{k, i}^{0}\right) \leq 1 .
\end{aligned}
$$

Remark 4.2 (Monotony of the scheme). Under the assumptions of Theorem 4.1, we have

$$
\begin{aligned}
\left|v_{+, i}^{n}-v_{-, i}^{n}\right| & \leq\left|v_{+, i}^{n}-\rho_{+}\left(x_{i}, t_{n}\right)\right|+\left|\rho_{+}\left(x_{i}, t_{n}\right)-\rho_{-}\left(x_{i}, t_{n}\right)\right|+\left|\rho_{-}\left(x_{i}, t_{n}\right)-v_{-, i}^{n}\right| \\
& \leq 2+M
\end{aligned}
$$

where we have used Theorem 3.2 (iv) for the second term. We then deduce that the discrete velocity is uniformly bounded:

$$
C_{k}^{\Delta, \operatorname{Loc}}[v] \leq\|a\|_{L^{\infty}(0, T)}+M+2=L_{1} .
$$

Then, one can show that the scheme is monotone in the following sense: let $v$ and $w$ be two discrete functions such that $v_{i}^{n} \leq w_{i}^{n}$; then

$$
S^{k}\left(v^{n}\right)\left(x_{i}\right) \leq S^{k}\left(w^{n}\right)\left(x_{i}\right), \quad \text { for } k \in\{+,-\} .
$$

For the proof of Theorem 4.1, we need the following lemma: 
Lemma 4.3. If $v_{i}^{n}$ is the numerical solution of (4.26)-(4.27), then

$$
-K t_{n}-\mu^{0} \leq \rho^{0}\left(x_{i}\right)-v\left(x_{i}, t_{n}\right) \leq K t_{n}+\mu^{0}
$$

where $K=2\left(\left\|P_{+}^{0}-P_{-}^{0}\right\|_{L^{\infty}(\mathbb{R})}+\|a\|_{L^{\infty}(0, T)}\right) \max _{k \in\{+,-\}}\left\|D \rho_{k}^{0}\right\|_{L^{\infty}(\mathbb{R})}$ and

$$
\mu^{0}=\max _{k \in\{+,-\}} \sup _{\Xi}\left|\rho_{k}^{0}\left(x_{i}\right)-v_{k, i}^{0}\right| \geq 0 .
$$

Proof of Lemma 4.3. To prove this, we set $w_{ \pm}\left(x_{i}, t_{n}\right)=\rho_{ \pm}^{0}\left(x_{i}\right)-K t_{n}-\mu^{0}$ and we show that for $K$ large enough $w$ is a discrete sub-solution. Indeed, we have

$$
\begin{aligned}
& w_{ \pm, i}^{n+1}-\left(S^{ \pm} w^{n}\right)_{i} \\
= & -K \Delta t-\Delta t C_{ \pm}^{\Delta, \operatorname{Loc}}\left[\rho^{0}\right]\left(x_{i}, t_{n}\right) E^{\operatorname{sgn}\left(C_{ \pm}^{\Delta, \mathrm{Loc}}\left[\rho^{0}\right]\left(x_{i}, t_{n}\right)\right)}\left(D^{+} \rho_{ \pm}^{0}\left(x_{i}\right), D^{-} \rho_{ \pm}^{0}\left(x_{i}\right)\right) \\
= & -\Delta t\left(K \mp\left(\rho_{+}^{0}\left(x_{i}\right)-\rho_{-}^{0}\left(x_{i}\right)+a^{\Delta}\left(t_{n}\right)\right)\right. \\
& \left.E^{\operatorname{sgn}\left(C_{ \pm}^{\Delta, \mathrm{Loc}}\left[\rho^{0}\right]\left(x_{i}, t_{n}\right)\right)}\left(D^{+} \rho_{ \pm}^{0}\left(x_{i}\right), D^{-} \rho_{ \pm}^{0}\left(x_{i}\right)\right)\right) \\
\leq & -\Delta t\left(K-2\left(\left\|P_{+}^{0}-P_{-}^{0}\right\|_{L^{\infty}(\mathbb{R})}+\|a\|_{L^{\infty}(0, T)}\right) \max _{k \in\{+,-\}}\left\|D \rho_{k}^{0}\right\|_{L^{\infty}(\mathbb{R})}\right)
\end{aligned}
$$

where $C_{k}^{\Delta, \text { Loc }}[w]\left(x_{i}, t_{n}\right)$ is defined in (4.28) and $\operatorname{sgn}(f)$ is the sign of $f$.

So, for every $K \geq 2\left(\left\|P_{+}^{0}-P_{-}^{0}\right\|_{L^{\infty}(\mathbb{R})}+\|a\|_{L^{\infty}(0, T)}\right) \max _{k \in\{+,-\}}\left\|D \rho_{k}^{0}\right\|_{L^{\infty}(\mathbb{R})}, w$ is a discrete sub-solution. Moreover,

$$
w_{ \pm, i}^{0}\left(x_{i}\right)=\rho_{ \pm}^{0}\left(x_{i}\right)-\mu^{0} \leq v_{ \pm}^{0}\left(x_{i}\right) .
$$

Using the monotony of the scheme, we deduce $w_{i}^{n} \leq v_{i}^{n}$ and so

$$
\rho^{0}\left(x_{i}\right)-v_{i}^{n} \leq K t_{n}+\mu_{0} .
$$

The lower bound is proved similarly.

We now give the proof of Theorem 4.1

Proof of Theorem 4.1. The proof is an adaptation for systems of the one of Crandall Lions [10, revisited by Alvarez et al. [2]. The proof splits into three steps. We denote throughout by $K$ various constants depending only on $\left\|P_{+}^{0}-P_{-}^{0}\right\|_{L^{\infty}(\mathbb{R})}$, $\max _{k \in\{+,-\}}\left\|D \rho_{k}^{0}\right\|_{L^{\infty}(\mathbb{R})}$ and $\|a\|_{W^{1, \infty(0, T)}}$.

We first assume that

$$
\rho^{0}\left(x_{i}\right) \geq v_{i}^{0}
$$

and we set

$$
\mu^{0}=\max _{k \in\{+,-\}} \sup _{\Xi}\left|\rho_{k}^{0}\left(x_{i}\right)-v_{k, i}^{0}\right| \geq 0 .
$$

We set a few notations. We put

$$
\mu=\max _{k \in\{+,-\}} \sup _{\Xi_{T}}\left(\rho_{k}\left(x_{i}, t_{n}\right)-v_{k, i}^{n}\right) .
$$

For every $0<\alpha \leq 1,0<\varepsilon \leq 1$ and $\sigma>0$, we set

$$
M_{\sigma}^{\alpha, \varepsilon}=\sup _{\mathbb{R} \times[0, T] \times \Xi_{T} \times\{+,-\}} \Psi_{\sigma}^{\alpha, \varepsilon}\left(x, t, x_{i}, t_{n}, k\right),
$$

with

$\Psi_{\sigma}^{\alpha, \varepsilon}\left(x, t, x_{i}, t_{n}, k\right)=\rho_{k}(x, t)-v_{k}\left(x_{i}, t_{n}\right)-\frac{\left|x-x_{i}\right|^{2}}{2 \varepsilon}-\frac{\left|t-t_{n}\right|^{2}}{2 \varepsilon}-\sigma t-\alpha|x|^{2}-\alpha\left|x_{i}\right|^{2}$. 
We shall drop the super and subscripts on $\Psi$ when no ambiguity arises concerning the value of the parameter.

Since $\rho^{0}$ is Lipschitz continuous and $T \leq 1$, we have by (3.22)

$$
\left|\rho_{ \pm}(x, t)\right| \leq K(1+|x|) .
$$

Moreover, by Lemma 4.3 we have

$$
\begin{aligned}
\left|v_{ \pm}\left(x_{i}, t_{n}\right)\right| & \leq\left|v_{ \pm}\left(x_{i}, t_{n}\right)-\rho_{ \pm}^{0}\left(x_{i}\right)\right|+\left|\rho_{ \pm}^{0}\left(x_{i}\right)\right| \\
& \leq K t_{n}+K\left(1+\left|x_{i}\right|\right) \\
& \leq K\left(1+\left|x_{i}\right|\right) .
\end{aligned}
$$

We then deduce that $\Psi$ achieves its maximum at some point that we denote by $\left(x^{*}, t^{*}, x_{i}^{*}, t_{n}^{*}, k^{*}\right)$.

\section{Step 1: Estimates for the maximum point of $\Psi$.}

The maximum point of $\Psi$ enjoys the following estimates:

$$
\left|x^{*}-x_{i}^{*}\right| \leq K \varepsilon, \quad\left|t^{*}-t_{n}^{*}\right| \leq(K+2 \sigma) \varepsilon .
$$

Indeed, by inequality $\Psi\left(x^{*}, t^{*}, x_{i}^{*}, t_{n}^{*}, k^{*}\right) \geq \Psi\left(0,0,0,0, k^{*}\right) \geq 0$, we obtain

$$
\begin{aligned}
\alpha\left|x^{*}\right|^{2}+\alpha\left|x_{i}^{*}\right|^{2} & \leq \rho_{k^{*}}\left(x^{*}, t^{*}\right)-v_{k^{*}}\left(x_{i}^{*}, t_{n}^{*}\right) \\
& \leq K\left(1+\left|x^{*}\right|+\left|x_{i}^{*}\right|\right) \leq K+\frac{K^{2}}{\alpha}+\frac{\alpha}{2}\left|x^{*}\right|^{2}+\frac{\alpha}{2}\left|x_{i}^{*}\right|^{2} .
\end{aligned}
$$

This implies (4.39), since $\alpha \leq 1$.

The first bound of (4.40) follows from the Lipschitz in space regularity of $\rho$ (see Theorem 3.2 (iii)), from the inequality $\Psi\left(x^{*}, t^{*}, x_{i}^{*}, t_{n}^{*}, k^{*}\right) \geq$ $\Psi\left(x_{i}^{*}, t^{*}, x_{i}^{*}, t_{n}^{*}, k^{*}\right)$ and from (4.39). Indeed, this implies

$$
\begin{aligned}
\frac{\left|x^{*}-x_{i}^{*}\right|^{2}}{2 \varepsilon} & \leq \rho_{k^{*}}\left(x^{*}, t^{*}\right)-\rho_{k^{*}}\left(x_{i}^{*}, t^{*}\right)-\alpha\left|x^{*}\right|^{2}+\alpha\left|x_{i}^{*}\right|^{2} \\
& \leq K\left|x^{*}-x_{i}^{*}\right|+\alpha\left|x^{*}-x_{i}^{*}\right|\left(\left|x^{*}\right|+\left|x_{i}^{*}\right|\right) \leq K\left|x^{*}-x_{i}^{*}\right| .
\end{aligned}
$$

The second bound of (4.40) is obtained in the same way, using the inequality $\Psi\left(x^{*}, t^{*}, x_{i}^{*}, t_{n}^{*}, k^{*}\right) \geq \Psi\left(x^{*}, t_{n}^{*}, x_{i}^{*}, t_{n}^{*}, k^{*}\right)$ and Theorem 3.2 (iii).

Step 2 : A better estimate for the maximum point of $\Psi$.

Inequality (4.39) can be strengthened to

$$
\alpha\left|x^{*}\right|^{2}+\alpha\left|x_{i}^{*}\right|^{2} \leq K .
$$

Indeed, using the Lipschitz regularity of $\rho$, the inequality

$$
\Psi\left(x^{*}, t^{*}, x_{i}^{*}, t_{n}^{*}, k^{*}\right) \geq \Psi\left(0,0,0,0, k^{*}\right)
$$

and equations (3.22), (4.34) and (4.40), yields

$$
\begin{aligned}
\alpha\left|x^{*}\right|^{2}+\alpha\left|x_{i}^{*}\right|^{2} & \leq \rho_{k^{*}}\left(x^{*}, t^{*}\right)-v_{k^{*}}\left(x_{i}^{*}, t_{n}^{*}\right)+\rho\left(x_{i}^{*}, 0\right)-\rho\left(x_{i}^{*}, 0\right) \\
& \leq K\left(\left|x^{*}-x_{i}^{*}\right|+t^{*}\right)+K t_{n}^{*}+\mu^{0} \leq K .
\end{aligned}
$$




\section{Step 3 : Upper bound of $\mu$.}

We have the bound $\mu \leq K \sqrt{T}(\Delta x+\Delta t)^{\frac{1}{2}}+\mu^{0}$ if $\Delta x+\Delta t \leq \frac{1}{K^{2}}$.

First, we claim that for $\sigma$ large enough, we have either $t^{*}=0$ or $t_{n}^{*}=$ 0 . Suppose the contrary. Then the function $(x, t) \mapsto \Psi\left(x, t, x_{i}^{*}, t_{n}^{*}, k^{*}\right)$ achieves its maximum at a point of $\mathbb{R} \times(0, T]$. Using the fact that $\rho$ is a sub-solution of the continuous problem, we obtain the inequality

$$
\sigma+p_{t}^{*} \leq-k^{*}\left(\rho_{+}-\rho_{-}+a\left(t^{*}\right)\right)\left|p_{x}^{*}+2 \alpha x^{*}\right|
$$

with $p_{t}^{*}=\frac{t^{*}-t_{n}^{*}}{\varepsilon}, p_{x}^{*}=\frac{x^{*}-x_{i}^{*}}{\varepsilon}$.

Since $t_{n}^{*}>0$, we also have $\Psi\left(x^{*}, t^{*}, x_{i}^{*}, t_{n}^{*}, k^{*}\right) \geq \Psi\left(x^{*}, t^{*}, x_{i}, t_{n}^{*}-\right.$ $\left.\Delta t, k^{*}\right)$. This implies

$$
v_{k^{*}}\left(\cdot, t_{n}^{*}-\Delta t\right) \geq \varphi\left(\cdot, t_{n}^{*}-\Delta t\right)+v_{k^{*}}\left(x_{i}^{*}, t_{n}^{*}\right)-\varphi\left(x_{i}^{*}, t_{n}^{*}\right)
$$

for $\varphi\left(x_{i}, t_{n}\right)=-\frac{\left|x^{*}-x_{i}\right|^{2}}{2 \varepsilon}-\frac{\left|t^{*}-t_{n}\right|^{2}}{2 \varepsilon}-\alpha\left|x_{i}\right|^{2}$. Using the fact that the scheme is monotone and commutes with the addition of constants, yields

$$
\begin{aligned}
v_{k^{*}}\left(x_{i}^{*}, t_{n}^{*}\right)= & S^{k^{*}}\left(v_{k^{*}}\left(\cdot, t_{n}^{*}-\Delta t\right)\right)\left(x_{i}^{*}\right) \\
\geq & \varphi\left(x_{i}^{*}, t_{n}^{*}-\Delta t\right)+v_{k^{*}}\left(x_{i}^{*}, t_{n}^{*}\right)-\varphi\left(x_{i}^{*}, t_{n}^{*}\right) \\
& +\Delta t\left(c_{k^{*}}^{\Delta, \text { Loc }}[v]\left(x_{i}^{*}, t_{n}^{*}\right)\right) E^{l^{*}}\left(D^{+} \varphi\left(x_{i}^{*}, t_{n}^{*}-\Delta t\right), D^{-} \varphi\left(x_{i}^{*}, t_{n}^{*}-\Delta t\right)\right)
\end{aligned}
$$

where $l^{*}=\operatorname{sgn}\left(c_{k^{*}}^{\Delta, \text { Loc }}[v]\left(x_{i}^{*}, t_{n}^{*}\right)\right)$. We set

$$
c[v]=-c_{k^{*}}^{\Delta, \operatorname{Loc}}[v]\left(x_{i}^{*}, t_{n}^{*}\right), \quad c[\rho]=k^{*}\left(\rho_{+}\left(x^{*}, t^{*}\right)-\rho_{-}\left(x^{*}, t^{*}\right)+a\left(t^{*}\right)\right) .
$$

We then obtain the super-solution inequality:

$$
\frac{\varphi\left(x_{i}^{*}, t_{n}^{*}\right)-\varphi\left(x_{i}^{*}, t_{n}^{*}-\Delta t\right)}{\Delta t} \geq-c[v] E^{l^{*}}\left(D^{+} \varphi\left(x_{i}^{*}, t_{n}^{*}-\Delta t\right), D^{-} \varphi\left(x_{i}^{*}, t_{n}^{*}-\Delta t\right)\right) .
$$

Straightforward computations of the discrete derivative of $\varphi$ yield

$$
p_{t}^{*}+\frac{\Delta t}{2 \varepsilon} \geq-c[v] E^{l^{*}}\left(p_{x}^{*}-\frac{\Delta x}{2 \varepsilon}-\alpha\left(2 x_{i}^{*}+\Delta x\right), p_{x}^{*}+\frac{\Delta x}{2 \varepsilon}-\alpha\left(2 x_{i}^{*}-\Delta x\right)\right) .
$$

Subtracting the above inequality to (4.42), we deduce

$$
\begin{aligned}
\sigma & \leq \frac{\Delta t}{2 \varepsilon}-c[\rho]\left|p_{x}^{*}+2 \alpha x^{*}\right|+c[v] E^{l^{*}}\left(p_{x}^{*}-\frac{\Delta x}{2 \varepsilon}-\alpha\left(2 x_{i}^{*}+\Delta x\right), p_{x}^{*}+\frac{\Delta x}{2 \varepsilon}-\alpha\left(2 x_{i}^{*}-\Delta x\right)\right) \\
& \leq \frac{\Delta t}{2 \varepsilon}-(c[\rho]-c[v])\left|p_{x}^{*}\right|+\alpha K\left|x^{*}\right| \\
& +|c[v]|\left|E^{l^{*}}\left(p_{x}^{*}-\frac{\Delta x}{2 \varepsilon}-\alpha\left(2 x_{i}^{*}+\Delta x\right), p_{x}^{*}+\frac{\Delta x}{2 \varepsilon}-\alpha\left(2 x_{i}^{*}-\Delta x\right)\right)-E^{l^{*}}\left(p_{x}^{*}, p_{x}^{*}\right)\right| \\
& \leq \frac{\Delta t}{2 \varepsilon}-(c[\rho]-c[v])\left|p_{x}^{*}\right|+K \alpha\left|x^{*}\right|+K \frac{\Delta x}{\varepsilon}+2 \alpha K\left|x_{i}^{*}\right|+2 \alpha K \Delta x
\end{aligned}
$$

where we have used, for the second line, the fact that

$$
c[\rho] \leq M+2 B_{0}\left(M+\|a\|_{L^{\infty}(0, T)}\right) T \leq K
$$

with $M=\left\|P_{+}^{0}-P_{-}^{0}\right\|_{L^{\infty}(\mathbb{R})}$ and $B_{0}=\max _{k \in\{+,-\}}\left\|D \rho_{k}^{0}\right\|_{L^{\infty}(\mathbb{R})}$ (see Theorem [3.2). Now, since $\rho_{k^{*}}\left(x^{*}, t^{*}\right)-v_{k^{*}}\left(x_{i}^{*}, t_{n}^{*}\right)=\max _{k \in\{+,-\}}\left(\rho_{k}\left(x^{*}, t^{*}\right)-\right.$ 
$\left.v_{k}\left(x_{i}^{*}, t_{n}^{*}\right)\right) \geq 0$, by Lemma 3.1 we obtain

$$
\begin{aligned}
-(c[\rho]-c[v])\left|p_{x}^{*}\right|= & -k^{*}\left(\rho_{+}\left(x^{*}, t^{*}\right)-\rho_{-}\left(x^{*}, t^{*}\right)+a\left(t^{*}\right)\right)\left|p_{x}^{*}\right| \\
& +k^{*}\left(v_{+}\left(x_{i}^{*}, t_{n}^{*}\right)-v_{-}\left(x_{i}^{*}, t_{n}^{*}\right)+a\left(t^{*}\right)\right)\left|p_{x}^{*}\right| \\
& +k^{*}\left(a^{\Delta}\left(t_{n}^{*}\right)-a\left(t^{*}\right)\right)\left|p_{x}^{*}\right| \\
\leq & \left|a^{\Delta}\left(t_{n}^{*}\right)-a\left(t^{*}\right)\right| p_{x}^{*}|\leq K| t_{n}^{*}-t^{*}|| p_{x}^{*} \mid
\end{aligned}
$$

where we have used (4.29). This implies

$$
\begin{aligned}
\sigma & \leq \frac{\Delta t}{2 \varepsilon}+K\left|t^{*}-t_{n}^{*}\right|\left|p_{x}^{*}\right|+K \alpha\left|x^{*}\right|+K \frac{\Delta x}{\varepsilon}+2 \alpha K\left|x_{i}^{*}\right|+2 \alpha K \Delta x \\
& \leq K \frac{\Delta x+\Delta t}{\varepsilon}+K \alpha^{1 / 2}+K \varepsilon .
\end{aligned}
$$

Putting

$$
\sigma^{*}=\sigma^{*}(\Delta x+\Delta t, \varepsilon, \alpha)=K \frac{\Delta x+\Delta t}{\varepsilon}+K\left(\alpha^{1 / 2}+\varepsilon\right),
$$

we therefore conclude that we must have $t^{*}=0$ or $t_{n}^{*}=0$ provided $\sigma \geq \sigma^{*}$. Whenever $t^{*}=0$, we deduce from Lemma 4.3 and from (4.40) that

$$
\begin{aligned}
M_{\sigma}^{\alpha, \varepsilon} & =\Psi\left(x^{*}, 0, x_{i}^{*}, t_{n}^{*}, k^{*}\right) \leq \rho_{k^{*}}^{0}\left(x^{*}\right)-v_{k^{*}}\left(x_{i}^{*}, t_{n}^{*}\right) \\
& \leq \rho_{k^{*}}^{0}\left(x^{*}\right)-\rho_{k^{*}}^{0}\left(x_{i}^{*}\right)+K t_{n}^{*}+\mu^{0} \\
& \leq K\left(\left|x^{*}-x_{i}^{*}\right|+t_{n}^{*}\right)+\mu^{0} \leq K(1+\sigma) \varepsilon+\mu^{0} .
\end{aligned}
$$

Similarly, whenever $t_{n}^{*}=0$, we deduce from the Lipschitz regularity of $\rho$ and from (4.40) that

$$
\begin{aligned}
M_{\sigma}^{\alpha, \varepsilon} & =\Psi\left(x^{*}, t^{*}, x_{i}^{*}, 0, k^{*}\right) \leq \rho_{k^{*}}\left(x^{*}, t^{*}\right)-v_{k^{*}}\left(x_{i}^{*}, 0\right) \\
& \leq K\left(\left|x^{*}-x_{i}^{*}\right|+t^{*}\right)+\mu^{0} \leq K(1+\sigma) \varepsilon+\mu^{0} .
\end{aligned}
$$

To sum up, we have shown that

$$
M_{\sigma}^{\alpha, \varepsilon} \leq K(1+\sigma) \varepsilon+\mu^{0} \leq K \varepsilon+\mu^{0}
$$

provided $\sigma^{*}=K \frac{\Delta x+\Delta t}{\varepsilon}+K\left(\alpha^{1 / 2}+\varepsilon\right) \leq \sigma \leq 1$. We then deduce that, for every $\left(x_{i}, t_{n}\right)$ and for every $k$, we have

$$
\begin{aligned}
& \rho_{k}\left(x_{i}, t_{n}\right)-v_{k}\left(x_{i}, t_{n}\right)-\left(K \frac{\Delta x+\Delta t}{\varepsilon}+K\left(\alpha^{1 / 2}+\varepsilon\right)\right) T-2 \alpha\left|x_{i}\right|^{2} \\
& \leq M_{\sigma}^{\alpha, \varepsilon} \leq K \varepsilon+\mu^{0} .
\end{aligned}
$$

Sending $\alpha \rightarrow 0$, taking the supremum over $\left(x_{i}, t_{n}\right)$, the maximum over $k$ and choosing $\varepsilon=T^{1 / 2}(\Delta x+\Delta t)^{1 / 2}$, we conclude that

$$
\begin{aligned}
\max _{k \in\{+,-\}} \sup _{\Xi_{T}}\left(\rho_{k}\left(x_{i}, t_{n}\right)-v_{k, i}^{n}\right)= & \mu \leq K(\Delta x+\Delta t)^{1 / 2} \sqrt{T} \\
& +\max _{k \in\{+,-\}} \sup _{\Xi}\left(\rho_{k}^{0}\left(x_{i}\right)-v_{k, i}^{0}\right),
\end{aligned}
$$

provided that $\Delta x, \Delta t$ are small enough, $T \leq 1, \mu_{0} \leq 1$, and (4.36) is assumed. 
In the general case, we consider $\bar{\rho}=\rho+\mu^{1}$ with $\mu^{1}=\max _{k \in\{+,-\}} \sup _{\Xi}\left(v_{k, i}^{0}-\rho_{k}^{0}\left(x_{i}\right)\right)$. We remark that $\bar{\rho}$ is solution of (3.19) and satisfies $\bar{\rho}^{0}\left(x_{i}\right) \geq v_{i}^{0}$. Then (4.43) is true with $\bar{\rho}$ in place of $\rho$, i.e.

$$
\begin{aligned}
\max _{k \in\{+,-\}} \sup _{\Xi_{T}}\left(\rho_{k}\left(x_{i}, t_{n}\right)+\mu^{1}-v_{k, i}^{n}\right) \leq & K(\Delta x+\Delta t)^{1 / 2} \sqrt{T} \\
& +\max _{k \in\{+,-\}} \sup _{\Xi}\left(\rho_{k}^{0}\left(x_{i}\right)+\mu^{1}-v_{k, i}^{0}\right),
\end{aligned}
$$

which still implies (4.43) with $\max _{k \in\{+,-\}} \sup _{\Xi}\left|\rho_{k}^{0}\left(x_{i}\right)-v_{k, i}^{0}\right|$.

The lower bound for the error estimate is obtained by exchanging $\rho$ and $v$. As the proof is similar to the above, we omit it.

4.2. Approximation of the non-local system. To solve numerically the nonlocal system (1.1)-(1.2), we use the finite difference scheme (1.3)-(1.4)-(1.5). We also assume the CFL condition (1.8). In particular, using Proposition [3.5, we deduce that the CFL condition (4.33) is satisfied uniformly for all $a$ defined by (1.5) because

$$
\|a[\rho]\|_{L^{\infty}(0, T)} \leq M+\|L\|_{L^{\infty}(0, T)}
$$

and so $L_{1} \leq L_{2}$.

Let $\bar{T} \geq 0$ which will be chosen later. To prove our convergence result, we mimic the continuous case and we rewrite the scheme (1.3)-(1.4)-(1.5) as a fixed point. Before proving Theorem 1.3 we need to introduce some notations and lemmata. Defining $X_{T}^{1, \Delta}=\mathbb{R}^{\left\{0, \ldots, N_{T}\right\}}$ and $X_{T}^{2, \Delta}=\left(\mathbb{R}^{2}\right)^{\mathbb{Z} \times\left\{0, \ldots, N_{T}\right\}}$, the set of discrete functions defined on $\left\{0, \ldots, N_{T}\right\}$ and on the mesh $\Xi_{T}$ respectively, we denote by $G^{\Delta}: X_{\bar{T}}^{1, \Delta} \rightarrow X_{\bar{T}}^{2, \Delta}$ the operator that gives the discrete solution $v$ of the local problem (4.27) for a given velocity $a^{\Delta} \in X_{\bar{T}}^{1, \Delta}$, i.e.

$$
\left(G_{+}^{\Delta}\left(a^{\Delta}\right), G_{-}^{\Delta}\left(a^{\Delta}\right)\right)=G^{\Delta}\left(a^{\Delta}\right)=v .
$$

In particular, the scheme (1.3)-(1.4)-(1.5) can be rewritten as a fixed point of $G^{\Delta}\left(a^{\Delta}[\cdot]\right)$, i.e.

$$
v=G^{\Delta}\left(a^{\Delta}[v]\right)
$$

with $a^{\Delta}[\cdot]$ defined in (1.5). We set, for all $T \leq \bar{T}$,

$$
U_{T}^{\Delta}=\left\{\begin{array}{l|l}
w \in X_{T}^{2, \Delta}: & \begin{array}{l}
\sup _{\Xi_{T}}\left|D_{x}^{+} w_{ \pm}\right| \leq B_{0} \\
\sup _{\Xi_{T}}\left|D_{t}^{+} w_{ \pm}\right| \leq 2 B_{0}\left(2 M+\|L\|_{L^{\infty}(0, T)}+4\right), \\
\sup _{\Xi_{T}}\left|w_{+}-w_{-}\right| \leq M+2 \\
\Xi_{T}
\end{array}
\end{array}\right\}
$$

and

$$
V_{T}^{\Delta}=\left\{a^{\Delta} \in X_{T}^{1, \Delta}:\left|\sup _{\left\{0, \ldots, N_{T} \Delta t\right\}}\right| a^{\Delta} \mid \leq M+\|L\|_{L^{\infty}}+2\right\}
$$

where $M=\left\|P_{+}^{0}-P_{-}^{0}\right\|_{L^{\infty}(\mathbb{R})}$. One can easily check that

$$
\left\{(\rho)^{\Delta} \mid \rho \in U_{T}\right\} \subset U_{T}^{\Delta}
$$

and

$$
\left\{(a)^{\Delta}: \mid\|a\|_{L^{\infty}(0, T)} \leq M+\|L\|_{L^{\infty}(0, T)}\right\} \subset V_{T}^{\Delta}
$$

where $(f)^{\Delta}$ is the restriction to $\Xi_{T}$ of the continuous function $f$. We have the following lemma: 
Lemma 4.4. Assume that (1.8) holds. Then for all $T \leq \bar{T}$, the following inclusion holds:

(i) $a^{\Delta}\left[U_{T}^{\Delta}\right] \subset V_{T}^{\Delta}$,

(ii) $G^{\Delta}\left(V_{T}^{\Delta}\right) \subset U_{T}^{\Delta}$.

Proof of Lemma 4.4. The proof of (i) is just a simple computation. We prove (ii).

Let $a^{\Delta} \in V_{T}^{\Delta}$ and $v=G^{\Delta}\left(a^{\Delta}\right)$. We set $w\left(x_{i}, t_{n}\right)=v\left(x_{i+1}, t_{n}\right)-\Delta x B_{0}$. Then $w$ is still a solution of the discrete scheme (4.27) and satisfies $w^{0} \leq v^{0}$. Using the monotony of the scheme yields

$$
\frac{v_{ \pm}\left(x_{i+1}, t_{n}\right)-v_{ \pm}\left(x_{i}, t_{n}\right)}{\Delta x} \leq B_{0} .
$$

Using Theorem 3.2, we deduce

$$
\left|v_{+}-v_{-}\right| \leq M+2 .
$$

For the estimate in time, we have, using (4.44),

$$
\begin{aligned}
\left|\frac{v_{i}^{n+1}-v_{i}^{n}}{\Delta t}\right| & \leq 2 B_{0}\left|C_{k}^{\Delta, \text { Loc }}[v]\left(x_{i}, t_{n}\right)\right| \leq 2 B_{0}\left(M+2+\sup _{\left\{0, \ldots, N_{T} \Delta t\right\}}\left|a^{\Delta}\right|\right) \\
& \leq 2 B_{0}\left(2 M+\|L\|_{L^{\infty}(0, T)}+4\right) ;
\end{aligned}
$$

so $G^{\Delta}\left(V_{T}^{\Delta}\right) \subset U_{T}^{\Delta}$. This ends the proof of the lemma.

We now have to prove some consistency and stability results for the velocity $a^{\Delta}$ and for the operator $G^{\Delta}$.

Lemma 4.5 (Consistency for the discrete velocity $a^{\Delta}[\cdot]$ ). There is a constant $K=$ $2 B_{0}+M$ such that, for every mesh $\Delta$, for every $0 \leq T \leq \bar{T}$ and for $\rho \in U_{T}$, we have

$$
\sup _{\left\{0, \ldots, N_{T} \Delta t\right\}}\left|(a[\rho])^{\Delta}-a^{\Delta}\left[(\rho)^{\Delta}\right]\right| \leq K \Delta x
$$

where $(\rho)^{\Delta}$ is the restriction to $\Xi_{T}$ of the continuous function $\rho$ and $a[\cdot]$ is defined in (3.24).

Proof of Lemma 4.5. We set $\tilde{\rho}(x, t)=\rho_{+}(x, t)-\rho_{-}(x, t)$. The following holds:

$$
\begin{aligned}
& \left|a[\rho]\left(t_{n}\right)-a^{\Delta}\left[(\rho)^{\Delta}\right]\left(t_{n}\right)\right|=\left|\int_{0}^{1} \tilde{\rho}\left(x, t_{n}\right) d x-\sum_{i=0}^{N_{x}-1} \Delta x \tilde{\rho}\left(x_{i}, t_{n}\right)\right| \\
& \quad \leq \sum_{i=0}^{N_{x}-1}\left|\int_{i \Delta x}^{(i+1) \Delta x} \tilde{\rho}\left(x, t_{n}\right) d x-\Delta x \tilde{\rho}\left(x_{i}, t_{n}\right)\right|+\int_{N_{x} \Delta x}^{1} \tilde{\rho}\left(x, t_{n}\right) d x \\
& \quad \leq \Delta x \sum_{i=0}^{N_{x}-1} \sup _{[i \Delta x,(i+1) \Delta x]}\left|\tilde{\rho}\left(\cdot, t_{n}\right)-\tilde{\rho}\left(x_{i}, t_{n}\right)\right|+M \Delta x \\
& \quad \leq \Delta x\left(2 B_{0}+M\right) .
\end{aligned}
$$

We have the following lemma which proof is just a simple computation

Lemma 4.6 (Stability property of the velocity $a^{\Delta}[\cdot]$ ). For every mesh $\Delta$, for every $0 \leq T \leq \bar{T}$ and every $v_{1}, v_{2} \in U_{T}^{\Delta}$, the following holds:

$$
\sup _{\left\{0, \ldots, N_{T} \Delta t\right\}}\left|a^{\Delta}\left[v_{2}\right]-a^{\Delta}\left[v_{1}\right]\right| \leq 2 \max _{k \in\{+,-\}} \sup _{\Xi_{T}}\left|v_{2}-v_{1}\right| .
$$


Lemma 4.7 (Stability property of the operator $G^{\Delta}$ ). There is a constant $K=$ $2 B_{0}$ so that, for every mesh $\Delta$ satisfying the uniform CFL condition (1.8), for all $0 \leq T \leq \bar{T}$ and all $a_{1}^{\Delta}, a_{2}^{\Delta} \in V_{T}^{\Delta}$

$$
\max _{k \in\{+,-\}} \sup _{\Xi_{T}}\left|G_{k}^{\Delta}\left(a_{2}^{\Delta}\right)-G_{k}^{\Delta}\left(a_{1}^{\Delta}\right)\right| \leq K T \sup _{\left\{0, \ldots, N_{T} \Delta t\right\}}\left|a_{2}^{\Delta}-a_{1}^{\Delta}\right| .
$$

Proof of Lemma 4.7, We set $v_{i}=G^{\Delta}\left(a_{i}^{\Delta}\right)$. Using the fact that

$$
c_{1} E^{\operatorname{sgn}\left(c_{1}\right)}-c_{2} E^{\operatorname{sgn}\left(c_{2}\right)} \leq\left|c_{1}-c_{2}\right| \max \left(E^{+}, E^{-}\right)
$$

yields

$$
\begin{aligned}
& v_{2, k}^{n+1}-v_{2, k}^{n}+k \Delta t\left(v_{2,+}^{n}-v_{2,-}^{n}+a_{1}^{\Delta}\left(t_{n}\right)\right) E^{\operatorname{sgn}\left(v_{2,+}^{n}-v_{2,-}^{n}+a_{1}^{\Delta}\left(t_{n}\right)\right)}\left(D^{+} v_{2}^{n}, D^{-} v_{2}^{n}\right) \\
\leq & \Delta t\left|a_{2}^{\Delta}\left(t_{n}\right)-a_{1}^{\Delta}\left(t_{n}\right)\right| \max \left(E^{+}\left(D^{+} v_{2}^{n}, D^{-} v_{2}^{n}\right), E^{-}\left(D^{+} v_{2}^{n}, D^{-} v_{2}^{n}\right)\right) \\
\leq & 2 B_{0} \Delta t \sup _{\left\{0, \ldots, N_{T} \Delta t\right\}}\left|a_{1}^{\Delta}-a_{2}^{\Delta}\right| .
\end{aligned}
$$

Moreover, $\tilde{v}_{1}\left(x_{i}, t_{n}\right)=v_{1}\left(x_{i}, t_{n}\right)+2 B_{0} \sup _{\left\{0, \ldots, N_{T} \Delta t\right\}}\left|a_{1}^{\Delta}-a_{2}^{\Delta}\right| t_{n}$ is a solution of the same discrete equation. Since the scheme is monotone, one deduces that

$$
\max _{k \in\{+,-\}} \sup _{\Xi_{T}}\left|G_{k}^{\Delta}\left(a_{2}^{\Delta}\right)-G_{k}^{\Delta}\left(a_{1}^{\Delta}\right)\right| \leq 2 B_{0} T \sup _{\left\{0, \ldots, N_{T} \Delta t\right\}}\left|a_{2}^{\Delta}-a_{1}^{\Delta}\right| .
$$

This achieves the proof.

We now prove Theorem 1.3 .

Proof of Theorem 1.3. We use the main idea of Alvarez et al. [2].

We first assume that $T \geq \bar{T}$ and we set, for every $l \geq 1$,

$$
Q_{l}^{\Delta}=\Delta x \mathbb{Z} \times\left\{\Delta t N_{l}, \ldots, \Delta t N_{l+1}\right\}
$$

where $N_{l}$ is the integer part of $\frac{l \bar{T}}{\Delta t}$. As in the continuous case, on each interval $(l \bar{T},(l+1) \bar{T})$, we can iterate the process (since $\bar{T}$ depends only on $B_{0}$ which does not change with time) and construct, using a fixed point method (denoting by $G$ and $\left.G^{\Delta}\right), \rho$ and $v$ respectively as solutions of (1.1)-(1.2) and (1.3)-(1.4)-(1.5). We then have the inequality

$$
\begin{aligned}
\max _{k \in\{+,-\}} \sup _{Q_{l}^{\Delta}}\left|\rho_{k}-v_{k}\right| \leq & \max _{k \in\{+,-\}} \sup _{Q_{l}^{\Delta}}\left|G_{k, l}(a[\rho])-G_{k, l}^{\Delta}\left(a^{\Delta}[v]\right)\right| \\
\leq & \max _{k \in\{+,-\}} \sup _{Q_{l}^{\Delta}}\left|G_{k, l}(a[\rho])-G_{k, l}^{\Delta}\left((a[\rho])^{\Delta}\right)\right| \\
& +\max _{k \in\{+,-\}} \sup _{Q_{l}^{\Delta}}\left|G_{k, l}^{\Delta}\left((a[\rho])^{\Delta}\right)-G_{k, l}^{\Delta}\left(a^{\Delta}[v]\right)\right|,
\end{aligned}
$$

where the function $G_{l}^{\Delta}\left((a[\rho])^{\Delta}\right)=\left(G_{+, l}^{\Delta}\left((a[\rho])^{\Delta}\right), G_{-, l}^{\Delta}\left((a[\rho])^{\Delta}\right)\right)\left(\right.$ resp. $\left.G_{l}(a[\rho])\right)$ is simply the discrete solution of (4.26) (resp. the continuous solution of (1.1)) with the velocity $a[\rho]$ and initial condition $v^{N_{l}}$ (resp. $\rho\left(\cdot, N_{l} \mathcal{D} t\right)$ ). From Theorem 4.1, 
we then deduce

$$
\begin{aligned}
& \max _{k \in\{+,-\}} \sup _{Q_{l}^{\Delta}}\left|G_{k, l}(a[\rho])-G_{k, l}^{\Delta}\left((a[\rho])^{\Delta}\right)\right| \\
& \quad \leq K \sqrt{\bar{T} \Delta x}+\max _{k \in\{+,-\} \Delta x \mathbb{Z} \times N_{l} \Delta t}\left|\rho_{k}-v_{k}\right| \\
& \quad \leq l K \sqrt{\bar{T} \Delta x}+\max _{k \in\{+,-\}} \sup _{\Xi}\left|\rho_{k}^{0}-v_{k}^{0}\right| .
\end{aligned}
$$

For the second term, we use Lemmata $4.5,4.6$ and 4.7 to obtain

$$
\begin{aligned}
& \max _{k \in\{+,-\}} \sup _{Q_{l}^{\Delta}}\left|G_{k, l}^{\Delta}\left((a[\rho])^{\Delta}\right)-G_{k, l}^{\Delta}\left(a^{\Delta}[v]\right)\right| \\
\leq & K \bar{T} \sup _{\left\{N_{l} \Delta t, \ldots, N_{l+1} \Delta t\right\}}\left|(a[\rho])^{\Delta}-a^{\Delta}[v]\right| \\
\leq & K \bar{T} \sup _{\left\{N_{l} \Delta t, \ldots, N_{l+1 \Delta t}\right\}}\left(\left|(a[\rho])^{\Delta}-a^{\Delta}\left[(\rho)^{\Delta}\right]\right|+\left|a^{\Delta}\left[(\rho)^{\Delta}\right]-a^{\Delta}[v]\right|\right) \\
& \leq K \bar{T}\left(\Delta x+\max _{k \in\{+,-\}} \sup _{Q_{l}^{\Delta}}\left|\rho_{k}-v_{k}\right|\right) .
\end{aligned}
$$

This implies, for $\bar{T} \Delta x \leq 1$ and $K \bar{T}<1$,

$$
\max _{k \in\{+,-\}} \sup _{Q_{l}^{\Delta}}\left|\rho_{k}-v_{k}\right| \leq \frac{l K}{1-K \bar{T}} \sqrt{\bar{T} \Delta x}+\left(\max _{k \in\{+,-\}} \sup _{\Xi}\left|\rho_{k}^{0}-v_{k}^{0}\right|\right) \frac{1}{1-K \bar{T}} .
$$

We now take $\bar{l} \geq 1$ such that

$$
\bar{l} \bar{T} \leq T \leq(\bar{l}+1) \bar{T} .
$$

Then the following holds:

$$
\begin{aligned}
\max _{k \in\{+,-\}} \sup _{\Xi_{T}}\left|\rho_{k}-v_{k}\right| & \leq \frac{\bar{l} K}{1-K \bar{T}} \sqrt{\bar{T} \Delta x}+\left(\max _{k \in\{+,-\}} \sup _{\Xi}\left|\rho_{k}^{0}-v_{k}^{0}\right|\right) \frac{1}{1-K \bar{T}} \\
& \leq K T \sqrt{\Delta x}+K\left(\max _{k \in\{+,-\}} \sup _{\Xi}\left|\rho_{k}^{0}-v_{k}^{0}\right|\right), \text { if } T \geq \bar{T},
\end{aligned}
$$

where we have used the fact that $\bar{T}$ depends only on $B_{0}$.

Notice that, in the case where $T \leq \bar{T}$, from Theorem 4.1, (4.45) is replaced by

$$
\max _{k \in\{+,-\}} \sup _{\Xi_{T}}\left|G_{k}(a[\rho])-G_{k}^{\Delta}\left((a[\rho])^{\Delta}\right)\right| \leq K \sqrt{T \Delta x}+\max _{k \in\{+,-\}} \sup _{\Xi}\left|\rho_{k}^{0}-v_{k}^{0}\right|,
$$

and so we obtain

$$
\max _{k \in\{+,-\}} \sup _{\Xi_{T}}\left|\rho_{k}-v_{k}\right| \leq K \sqrt{T \Delta x}+K\left(\max _{k \in\{+,-\}} \sup _{\Xi}\left|\rho_{k}^{0}-v_{k}^{0}\right|\right), \text { if } T \leq \bar{T} .
$$

This ends the proof of the theorem. 


\section{NUMERICAL RESULTS}

In this section, we present some numerical simulations of the 1-D Groma-Balogh problem (1.1)-(1.2) discretized by the numerical scheme (1.3)-(1.4)-(1.5).

5.1. Numerical error estimate. Here, we show a numerical test in order to confirm our error estimate for the local system. Let us fix $L(t)=0$ even if it is not physicaly relevant, let us choose the following initial conditions: $\rho_{+}^{0}(x)=$ $-|x-1 / 2|+1 / 2$, and $\rho_{-}^{0}(x)=-|2 x-1|+1$ on $[0,1[$ (and extend it by periodicity on $\mathbb{R})$.

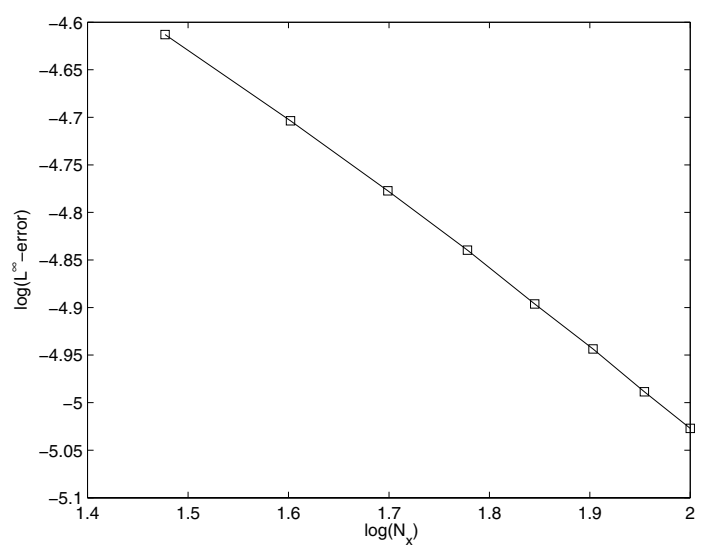

FigURE 3. $\log \left(L^{\infty}\right.$-error $)$ of $\left|u_{N_{T}}-u_{N_{T}-1}\right|$ versus $\log \left(N_{x}\right)$ at $T=\frac{1}{2}$

Figure 3 shows the behaviour of the $L^{\infty}$-error versus the discretization parameter $\Delta x$. The regression slope is close to 0.7 and the ideal regression is $\frac{1}{2}$. Hence, the behaviour of this error confirms that our error seems optimal.

5.2. Dislocations density dynamics. In this paragraph, we are interested in the evolution of dislocations densities for the 1-D Groma-Balogh model (1.1)-(1.2) under the uniformly applied shear stress $L(t)=3 t$.

In this simulation, we choose an example of concentrated dislocations densities, i.e. where dislocations densities are initially periodic, and equal to zero on some sub-intervals of $[0,1[$ (see Figure 4) ).

This initial condition means that there exists some regions without dislocations, and others with concentrated dislocations.

Intuitively, dislocations are intended to be uniformly distributed in the whole crystal as shown in (Figure 6) where finally a uniform distribution in all the crystal is observed, i.e. the density of dislocations becomes a constant.

We remark that when $L(t)$ is non-stationary, our system behaves as a diffusion equation (see [9] for further details), but evidently when $L(t)=0$, with the same initial condition, the system does not evolve. 


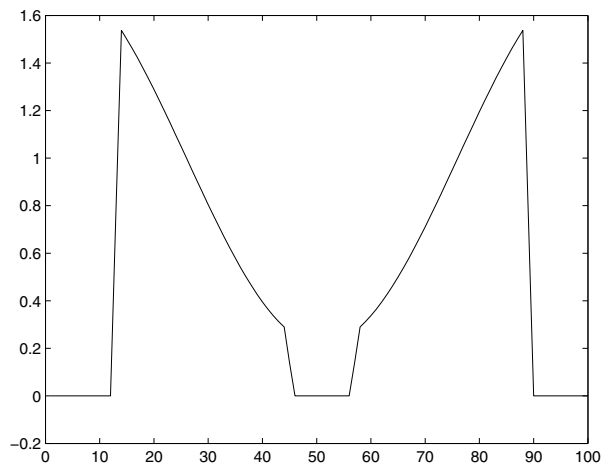

Figure 4. Dislocations density $\left(D \rho_{+}^{0}()=.D \rho_{-}^{0}().\right)$
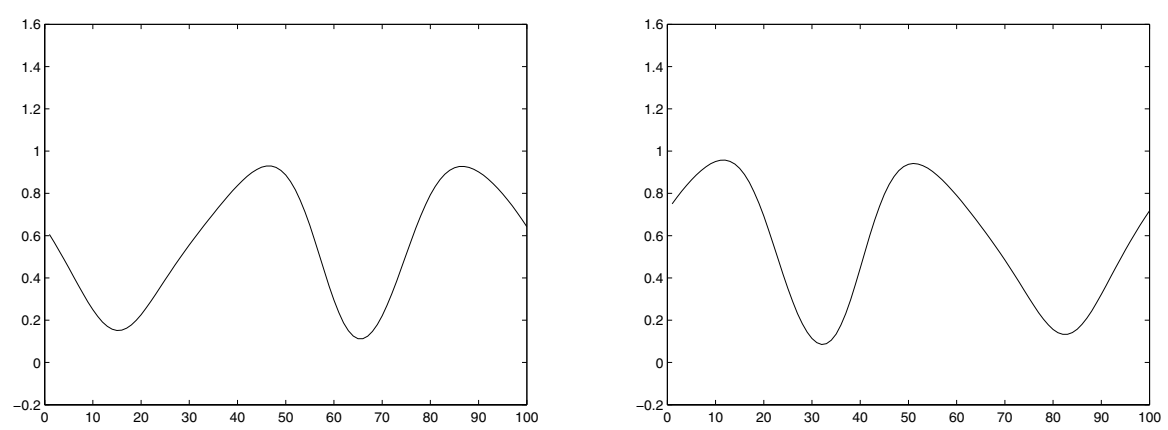

Figure 5. On the left: density $\left(D \rho_{+}\left(., \frac{1}{2}\right)\right)$; on the right: dislocations density $\left(D \rho_{-}\left(., \frac{1}{2}\right)\right)$

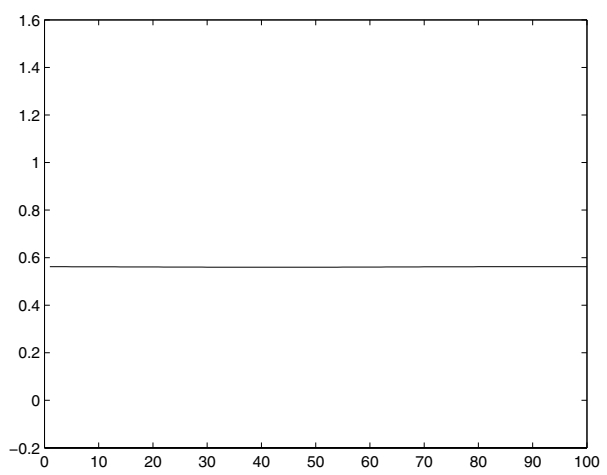

FiguRE 6. Dislocations density $\left(D \rho_{+}(., 3)=D \rho_{-}(., 3)\right)$ 


\section{ACKNOWLEDGEMENTS}

The authors would like to thank O. Alvarez, M. El Rhabi, C. Imbert, and R. Monneau for fruitful discussions in the preparation of this paper. This work was supported by the contract JC 1025 called "ACI jeunes chercheuses et jeunes chercheurs" of the French Ministry of Research (2003-2005).

\section{REFERENCES}

1. Olivier Alvarez, Pierre Cardaliaguet, and Régis Monneau, Existence and uniqueness for dislocation dynamics with nonnegative velocity, Interfaces and Free Boundaries 7 (2005), no. 4, 415-434. MR2191694 (2006i:35023)

2. Olivier Alvarez, Elisabetta Carlini, Régis Monneau, and Elisabeth Rouy, Convergence of a first order scheme for a non-local eikonal equation, IMACS Journal Applied Numerical Mathematics 56 (2006), 1136-1146. MR2244967 (2007d:65072)

3. _ A convergent scheme for a nonlocal Hamilton-Jacobi equation, modeling dislocation dynamics, Numerische Mathematik 104 (2006), no. 4, 413-572. MR.2249672

4. Olivier Alvarez, Philippe Hoch, Yann Le Bouar, and Régis Monneau, Résolution en temps court d'une équation de Hamilton-Jacobi non locale décrivant la dynamique d'une dislocation, C. R. Math. Acad. Sci. Paris 338 (2004), no. 9, 679-684. MR2065373 (2005b:35021)

5. Dislocation dynamics: short time existence and uniqueness of the solution, Archive for Rational Mechanics and Analysis 85 (2006), no. 3, 371-414.

6. Martino Bardi and Italo Capuzzo-Dolcetta, Optimal control and viscosity solutions of Hamilton-Jacobi-Bellman equations, Systems \& Control: Foundations \& Applications, Birkhäuser Boston, Inc., Boston, MA, 1997, With appendices by Maurizio Falcone and Pierpaolo Soravia. MR1484411 (99e:49001)

7. Guy Barles, Solutions de viscosité des équations de Hamilton-Jacobi, Mathématiques \& Applications (Berlin) [Mathematics \& Applications], vol. 17, Springer-Verlag, Paris, 1994. MR.1613876 (2000b:49054)

8. Guy Barles and Olivier Ley, Nonlocal first-order hamilton-jacobi equations modelling dislocations dynamics, Comm. Partial Differential Equations, 2006, 1191-1208. MR2254611

9. Ariela Briani et al., Homogenization in time for a model with dislocations densities, In preparation (2006).

10. M. G. Crandall and P.-L. Lions, Two approximations of solutions of Hamilton-Jacobi equations, Math. Comp. 43 (1984), no. 167, 1-19. MR744921 (86j:65121)

11. Michael G. Crandall, Hitoshi Ishii, and Pierre-Louis Lions, User's guide to viscosity solutions of second order partial differential equations, Bull. Amer. Math. Soc. (N.S.) 27 (1992), no. 1, 1-67. MR 1118699 (92j:35050)

12. Hans Engler and Suzanne M. Lenhart, Viscosity solutions for weakly coupled systems of Hamilton-Jacobi equations, Proc. London Math. Soc. (3) 63 (1991), no. 1, 212-240. MR 1105722 (93a:35024)

13. Nicolas Forcadel, Dislocations dynamics with a mean curvature term: short time existence and uniqueness, Preprint (2005).

14. I. Groma, Link between the microscopic and mesoscopic length-scale description of the collective behaviour of dislocations, Phys. Rev. B 56 (1997), 5807.

15. I. Groma and P. Balogh, Link between the individual and continuum approaches of the description of the collective behavior of dislocations, Mat. Sci. Eng. A234-236 (1997), 249-252.

16. Investigation of dislocation pattern formation in a two-dimensional self-consistent field approximation, Acta mater 47 (1999), no. 13, 3647-3654.

17. John Price Hirth and Jens Lothe, Theory of dislocations, Second edition, Krieger, Malabar, Florida, 1992.

18. Hitoshi Ishii, Perron's method for monotone systems of second-order elliptic partial differential equations, Differential Integral Equations 5 (1992), no. 1, 1-24. MR1141724 (92h:35071)

19. Hitoshi Ishii and Shigeaki Koike, Viscosity solutions for monotone systems of second-order elliptic PDEs, Comm. Partial Differential Equations 16 (1991), no. 6-7, 1095-1128. MR.1116855 (92h:35066)

20. Viscosity solutions of a system of nonlinear second-order elliptic PDEs arising in switching games, Funkcial. Ekvac. 34 (1991), no. 1, 143-155. MR1116886 (92h:35067) 
21. E. R. Jakobsen and K. H. Karlsen, Convergence rates for semi-discrete splitting approximations for degenerate parabolic equations with source terms, BIT 45 (2005), no. 1, 37-67. MR 2164225 (2006h:65135)

22. Espen Robstad Jakobsen, Kenneth Hvistendahl Karlsen, and Nils Henrik Risebro, On the convergence rate of operator splitting for Hamilton-Jacobi equations with source terms, SIAM J. Numer. Anal. 39 (2001), no. 2, 499-518 (electronic). MR1860266 (2002k:65138)

23. Suzanne M. Lenhart, Viscosity solutions for weakly coupled systems of first-order partial differential equations, J. Math. Anal. Appl. 131 (1988), no. 1, 180-193. MR934440 (89m:35025)

24. Suzanne M. Lenhart and Stavros A. Belbas, A system of nonlinear partial differential equations arising in the optimal control of stochastic systems with switching costs, SIAM J. Appl. Math. 43 (1983), no. 3, 465-475. MR700525 (85c:49029)

25. Suzanne M. Lenhart and Naoki Yamada, Viscosity solutions associated with switching game for piecewise-deterministic processes, Stochastics Stochastics Rep. 38 (1992), no. 1, 27-47. MR.1274894 (95b:49045)

26. Stanley Osher and James A. Sethian, Fronts propagating with curvature-dependent speed: algorithms based on Hamilton-Jacobi formulations, J. Comput. Phys. 79 (1988), no. 1, 12-49. MR:965860 (89h:80012)

27. Elisabeth Rouy and Agnès Tourin, A viscosity solutions approach to shape-from-shading, SIAM J. Numer. Anal. 29 (1992), no. 3, 867-884. MR1163361 (93d:65019)

28. Naoki Yamada, Viscosity solutions for a system of elliptic inequalities with bilateral obstacles, Funkcial. Ekvac. 30 (1987), no. 2-3, 417-425. MR927191 (88m:35061)

Cermics, Ecole des Ponts, Paristech 6 et 8 avenue Blaise Pascal, Cité Descartes, Champs-sur-Marne, 77455 Marne-la-Vallée Cedex 2

Cermics, Ecole des Ponts, Paristech 6 et 8 avenue Blaise Pascal, Cité Descartes, Champs-sur-Marne, 77455 Marne-la-Vallée Cedex 2 